\title{
LATTICE-ORDERED GROUPS WHOSE LATTICES DETERMINE THEIR ADDITIONS
}

\author{
PAUL F. CONRAD AND MICHAEL R. DARNEL
}

\begin{abstract}
In this paper it is shown that several large and important classes of lattice-ordered groups, including the free abelian lattice-ordered groups, have their group operations completely determined by the underlying lattices, or determined up to $l$-isomorphism.
\end{abstract}

\section{INTRODUCTION}

In the group of integers $\mathscr{Z}$ with the usual order $\leq, 1$ covers 0 . From this simple fact, it is easy to see that $(\mathscr{Z}, \leq)$ is a uniquely transitive chain as defined by Ohkuma [24] and that 1 is a singular element. Either property is enough to show that, having chosen 0 to be the identity of $\mathscr{Z}$, the usual addition is completely specified by the chain.

In this paper, we show that these properties are sufficiently general and powerful enough to prove that many large and familiar classes of lattice-ordered groups also have their group operations completely determined by the lattice and the choice of an identity. In particular, we will show

Theorem A. Every free abelian lattice-ordered group has a unique addition.

Theorem B. If $G$ is archimedean and if for any $0<g \in G$, there exists a singular element $s$ such that $0<s \leq g$, then $G$ has a unique addition.

Theorem C. For a Stone space $X$, any two additions on $\mathscr{D}(X)$, the l-group of continuous extended real-valued functions with densely open real support under pointwise order and addition, must be l-isomorphic.

Theorem $\mathbf{D}$. If $\Delta$ is a root system satisfying the descending chain condition and $G$ is a lattice-ordered group such that $\Sigma(\Delta, \mathscr{R}) \subseteq G \subseteq \mathscr{V}(\Delta, \mathscr{R})$, then any two abelian group operations on $(G, \leq)$ must be l-isomorphic.

We assume that the reader is familiar with the terms and theory of latticeordered groups as developed in [3] or [6]; our notation will be that of [6], as is common $^{1}$. The reader not familiar with the definitions of convex $l$-subgroups,

Received by the editors June 24, 1988 and, in revised form, January 3, 1990.

1980 Mathematics Subject Classification (1985 Revision). Primary 06F20.

This research was partially supported by a Summer Faculty Fellowship, 1987, from Indiana University at South Bend.

${ }^{1}$ A newly released book which the reader will find to be an excellent reference is Lattice-ordered Groups: An introduction, by Marlow Anderson and Todd Feil, Reidel, 1988. The terminology and notation there is that of [6]. 
prime and regular subgroups, polars, or plenary subsets of the regular subgroups can find these in either of the two references above. However, for the sake of the reader, we now include the following standard definitions. A regular convex $l$-subgroup is a convex $l$-subgroup maximal with respect to not including some element on the l-group; it is customary to denote the set of regular subgroups as $\Gamma(G)=\left\{G_{\lambda}: \lambda \in \Lambda\right\}$. Each regular subgroup $G_{\lambda}$ has a unique cover $G^{\lambda}$ minimal with respect to properly containing $G_{\lambda}$. If the lattice-ordered group is normal-valued, then $G^{\lambda} / G_{\lambda}$ is called a component of the lattice-ordered group and is order-isomorphic to a subgroup of the reals. Under containment, the set of regular subgroups $\Gamma(G)$ forms a root system: no two incomparable elements have a lower bound. A plenary subset of $\Gamma(G)$ is a dual ideal $\Delta$ such that $\bigcap_{\delta \in \Delta} G_{\delta}=(0)$.

For notational ease, we will use additive notation for all groups.

Following [4], an l-group $G$ is special-valued if every positive element is the join of a pairwise disjoint collection of special elements; this is equivalent to the set of special values forming a (necessarily minimal) plenary subset of the regular subgroups. We remind the reader that special-valued $l$-groups are normal-valued and completely distributive. If every positive element is the join of only finitely many disjoint special elements, the group is called finite-valued.

Finally, $(\mathscr{Z}, \leq),(\mathscr{Q}, \leq)$, and $(\mathscr{R}, \leq)$ will denote the integers, rationals, and reals, respectively, ordered in the usual fashion. $(\mathscr{Z}, \leq,+),(\mathscr{Q}, \leq,+)$, and $(\mathscr{R}, \leq,+)$ will denote these sets with the usual order and addition.

Now suppose that $(G, \leq)$ is a lattice admitting a group operation + such that $(G, \leq,+)$ is an $l$-group. Let 0 be the identity of $(G, \leq,+)$. For any element $g$, let $\tau_{g}$ be the right translation by $g$. Then $\tau_{g}$ is a lattice automorphism of $(G, \leq)$ and we can define a new operation $+_{g}$ by defining $x+g y$ to equal $\tau_{g}\left[\tau_{g}^{-1}(x)+\tau_{g}^{-1}(y)\right]$. Then clearly $\left(G, \leq, \tau_{g}\right)$ is an l-group, $l$-isomorphic to $(G, \leq,+)$, with identity $g$. So we can assume that any two group operations on a lattice $(G, \leq)$ have the same identity. Having assumed that and having chosen the identity element, we say that $(G, \leq)$ has unique addition if there is precisely one group operation + so that $(G, \leq,+)$ is an l-group. If $(G, \leq)$ has more than one such operation but has the additional property that for any two such operations + and $\oplus,(G, \leq,+)$ is $l$-isomorphic to $(G, \leq, \oplus)$, we say that $(G, \leq)$ essentially one addition. Note that if $(G, \leq)$ has essentially one addition and if $(G, \leq,+)$ and $(G, \leq, \oplus)$ are l-groups with common identity 0 , then the $l$-isomorphism $\tau$ between the two is just a lattice automorphism of $(G, \leq)$ preserving 0 , and that every other group operation is obtained from + in this fashion. So we will also restrict our attention to those lattice automorphisms of the lattice of an l-group that preserve the identity.

Finally, we wish to extend our thanks to a nameless referee who not only carefully read and analyzed our proofs but made many valuable suggestions as to how they might be improved or replaced by simpler ones.

\section{SUBGROUPS AND PROPERTIES INVARIANT UNDER GROUP OPERATIONS}

In this section, we collect for later use several results about which subgroups and properties of an arbitrary lattice-ordered group are invariant under all group operations compatible with the lattice and chosen identity. Our starting point is the following theorem, which gathers several results of Bixler and Darnel from [4]: 
Theorem 2.1. Let $(G, \leq,+)$ and $(G, \leq, \oplus)$ be l-groups on the lattice $(G, \leq)$ with the same identity. Let $S \subset G$. Then:

(a) $S$ is a polar of $(G,+)$ if and only if $S$ is a polar of $(G, \oplus)$.

(b) $S$ is a minimal prime subgroup of $(G,+)$ if and only if $S$ is a minimal prime subgroup of $(G, \oplus)$.

(c) $S$ is a cardinal summand of $(G,+)$ if and only if $S$ is a cardinal summand of $(G, \oplus)$.

(d) $S$ is the lex kernel of $(G,+)$ if and only if $S$ is the lex kernel of $(G, \oplus)$.

(e) $g \in G$ is special in $(G,+)$ if and only if $g$ is special in $(G, \oplus)$.

(f) $(G,+)$ is finite-valued or special-valued if and only if $(G, \oplus)$ is finitevalued or special-valued, respectively.

Note that the Boolean algebra of polars and the set of minimal prime subgroups of an $l$-group are completely determined by the lattice and the identity. However, this is not true for arbitrary convex $l$-subgroups. For as shown by Cantor, $(\mathscr{Q}, \leq)$ is characterized by being a dense unbounded countable totally ordered set. Let $H=\mathscr{Q} \leftarrow \mathscr{Z}$, ordered lexicographically with $(q, m) \geq(0,0)$ if $m>0$ or if $m=0$ and $q \geq 0$. On $H$, define $\left(q_{1}, m\right)\left(q_{2}, n\right)$ to equal $\left(q_{1}+2^{n} q_{2}, m+n\right)$. With this operation, $H$ is an $o$-group that as a chain is isomorphic to $(\mathscr{Q}, \leq)$. Note that in $H, \mathscr{Q} \times 0$ is a regular subgroup while $(\mathscr{Q}, \leq,+)$ has no proper convex subgroups. This shows that arbitrary prime subgroups need not remain subgroups under a new operation. This also shows that changing the group operation may not preserve archimedean or abelian properties.

Proposition 2.2. If $\tau$ is a lattice automorphism of $(G, \leq,+)$ preserving 0 , then for any $g \in G, \tau(g)=\tau\left(g^{+}\right)+\tau\left(-g^{-}\right)$.

Proof. $\tau(g)=a-b$, where $a \wedge b=0$. So $a=\tau(g) \vee 0=\tau(g) \vee \tau(0)=\tau(g \vee 0)=$ $\tau\left(g^{+}\right)$. Likewise, $b=[-\tau(g)] \vee 0=-[\tau(g) \wedge 0]=-[\tau(g \wedge 0)]=-\left[\tau\left(-g^{-}\right)\right]$.

Proposition 2.3. Let $(G, \leq,+)$ and $(G, \leq, \oplus)$ be l-groups. Then for any $g \in$ $G$, the mixed conjugate map: $x \rightarrow(g+x) \ominus g$ and the double inverse map: $x \rightarrow \ominus-x$ are lattice automorphisms preserving the identity.

In one way or another, most of our results come from the two lattice automorphisms presented above.

We now introduce a special kind of lattice automorphism of an l-group.

Definition. An identity-preserving lattice automorphism $\tau$ of an l-group is a p-permutation if whenever $|g| \wedge|h|=0,|g| \wedge|\tau(h)|=0$.

$P$-permutations are the lattice analogues of $p$-endomorphisms ( $l$-endomorphisms $\sigma$ where if $g \wedge h=0, g \wedge h \sigma=0$ ) and enjoy many of the same properties.

Theorem 2.4. For a lattice automorphism $\tau$, the following are equivalent:

(a) $\tau$ is a p-permutation.

(b) For all $g>0, g \wedge \tau(g)>0$.

(c) For all $g \in G, \tau(g) \in g^{\prime \prime}$.

(d) For every polar $P, \tau(P)=P$.

(e) For every minimal prime $M, \tau(M)=M$. 
Proof. (a) $\Rightarrow$ (b) If $g \wedge \tau(g)=0$, then $0=\tau(g) \wedge \tau(g)=\tau(g \wedge g)=\tau(g)$ and so $g=0$.

(b) $\Rightarrow$ (c) Assume $g>0$ but $\tau(g) \notin g^{\prime \prime}$. Then there exists $0<h \in g^{\prime}$ such that $\tau(g) \wedge h>0$ and so $g \wedge \tau^{-1}(h)>0$. But then $\left[g \wedge \tau^{-1}(h)\right] \wedge \tau\left[g \wedge \tau^{-1}(h)\right]>$ 0 , and so $0<g \wedge \tau^{-1}(h) \wedge \tau(g) \wedge h \leq g \wedge h=0$, a contradiction. To extend this to all $g$, note that $\tau(0)=0$ and apply Proposition 2.2.

$(\mathrm{c}) \Rightarrow(\mathrm{d}) \Rightarrow(\mathrm{e})$ Is easily adapted from similar results about $p$-endomorphisms.

(e) $\Rightarrow$ (a) Suppose $|g| \wedge|h|=0$ but $|g| \wedge|\tau(h)|>0$. There exists a minimal prime $M$ not containing $|g|$ and $|\tau(h)|$. Since $|g| \wedge|h|=0, h \in M$, contradicting $\tau(h) \notin M$.

Proposition 2.5. The p-permutations of an l-group $G$ form a normal subgroup of the group of all lattice automorphisms of $G$ that preserve the identity.

Proposition 2.6. If $\tau$ is a p-permutation of an l-group $G$, then $\sigma: x \rightarrow$ $-[\tau(-x)]$ is also a p-permutation of $G$.

Proposition 2.7. [4] If $(G, \leq,+)$ and $(G, \leq, \oplus)$ are l-groups, then the double inverse maps $x \rightarrow-\ominus x$ and $x \rightarrow \ominus-x$ are p-permutations.

Since inner automorphisms in general are not $p$-endomorphisms, mixed conjugate maps usually will not be $p$-permutations, even if one addition is abelian. However, mixed conjugate maps are $p$-permutations if one of the $l$-groups is archimedean.

Proposition 2.8. Suppose $(G, \leq,+)$ and $(G, \leq, \oplus)$ are both l-groups such that $(G, \leq,+)$ is archimedean. Then for any $0<g \in G$, the maps $x \rightarrow(g+x) \ominus g$ and $x \rightarrow(g \oplus x)-g$ are p-permutations.

Proof. Suppose there exists $h>0$ such that $[(g+h) \ominus g] \wedge h=0$; then $(g+h) \wedge$ $(h \oplus g)=g$ and so $h \leq g$. But now for any integer $n>0, n h \wedge[(g+h) \ominus g]=0$ and so $[-g+(n h \oplus g)] \wedge h=0$, implying $[-g+(n h \oplus g)] \wedge n h=0$. But then $n h \wedge[(g+n h) \ominus g]=0$ and so $n h \leq g$ for all integers $n$; so $h=0$.

From this, we get the following theorem.

Theorem 2.9. Suppose $(G, \leq,+)$ and $(G, \leq, \oplus)$ are l-groups.

(a) If $(G, \leq,+)$ is archimedean, then $(G, \leq, \oplus)$ is representable as a subdirect product of o-groups.

(b) If $(G, \leq,+)$ is hyperarchimedean and for each prime subgroup $M$, $(G,+) / M$ is either cyclic or real, then $(G, \leq, \oplus)$ is hyperarchimedean.

Proof. (a) For all $g>0$ and any minimal prime subgroup $M,(g+M) \ominus g=M$ and so $M+g=g+M=M \oplus g$. Likewise, for all $g \leq 0,(g+M) \ominus g=M$ and so $M$ is normal in $(G, \leq, \oplus)$. But then $(G, \leq, \oplus)$ is representable.

(b) Let $M$ be a minimal prime subgroup of $G$. By (a), $M$ is normal in $(G, \leq, \oplus)$ and for all $g \in G, M+g=M \oplus g$. Thus as ordered sets, $(G,+) / M=(G, \oplus) / M$, which is order isomorphic either to $\mathscr{Z}$ or to $\mathscr{R}$, both of which are Dedekind complete chains. Thus for every minimal prime $M,(G, \oplus) / M$ is an archimedean $O$-group and so $M$ is maximal as well. 
Our previous example shows that Theorem 2.9(a) is the best one can get for archimedean $l$-groups. It also shows that $p$-permutations need not preserve maximal convex $l$-subgroups. For in $(\mathscr{Q}, \leq,+)$, the map $g \rightarrow 2 g$ is a $p$ automorphism that determines a $p$-permutation on $H=\mathscr{Q} \overleftarrow{\times} \mathscr{Z}$. Since $(\mathscr{Q}, \leq$, $+)$ is archimedean, the corresponding $p$-permutation on $H$ cannot preserve $\mathscr{Q} \times\{0\}$.

As mentioned in the Introduction, $(\mathscr{Z}, \leq)$ is an example of a uniquely transitive chain: for any $a, b \in \mathscr{Z}$, there exists a unique order-preserving permutation $\tau$ such that $\tau(a)=b$. In [24], Ohkuma studied such chains, proving that every such chain $X$ is order isomorphic to a subgroup of $\mathscr{R}$ and that $\mathscr{A}(X)$, the group of order permutations of $X$, is just the right regular representation of this subgroup. He also proved that there exist $2^{2^{\omega}}$ nonisomorphic groups of this kind. We will call these groups Ohkuma groups and uniquely transitive chains Ohkuma chains. We now investigate some of the properties of Ohkuma chains.

Lemma 2.10. If $T$ is an Ohkuma chain and $C$ is a proper convex subset of $T$, then $C$ has no nontrivial order permutations.

Proof. Since $C$ is convex, any order permutation $\tau$ of $C$ can be extended to one of $T$ by defining the extension to be the identity on $T \backslash C$. But then $\tau$ must be the identity on $T$ and so on $C$.

Theorem 2.11. Let $T$ be a chain and $G$ be an l-group acting transitively on $T$ as a group of order-preserving permutations. Let $C$ be a convex subset of $T$ that is either a maximal Dedekind complete subchain or an Ohkuma chain. Then $C$ is an o-block [15, p. 12] of the action of $G$ on $T$. In particular, if $C$ is properly contained in $T$, there does not exist any order isomorphism of $T$ onto $C$.

Proof. The case when $C$ is a Dedekind complete chain was proven by Holland in [19].

So suppose $C$ is an Ohkuma chain. If $C$ is not an $o$-block, there exists $0<g \in G$ such that $g(C) \cap C \neq \varnothing$ but $g(C) \neq C$. Clearly $C \nsubseteq g(C)$ and $g(C) \nsubseteq C$. So there exists $c \in C$ such that $c<g(C)$ and there exists $d \in C$ such that $g(d)>C$.

Any order-preserving permutation of $C$ or of $g(C)$ can be extended to one of $S=C \cup g(C)$ and thus the group of order automorphisms of $S$ acts transitively on $S$. Since $C$ is an Ohkuma chain and is a proper initial segment of $S, S$ is not an Ohkuma chain. Thus there exist $a, b \in S$ and an order automorphism $\tau$ of $S$ such that $\tau(a)=a$ but $\tau(b) \neq b$. Assume $a \in C$. Now if for all $c \in C, \tau(c) \in C, \tau$ restricts to order permutations of both $C$ and $g(C)$ and so is the identity. So, without loss of generality, $b \in C$ but $\tau(b) \notin C$. But then $C \subset \tau(C)$, a contradiction since $C$ and $\tau(C)$ are Ohkuma chains.

Corollary 2.12. Let $(G, \leq,+)$ be an l-group and $M$ be a prime subgroup of $G$. Suppose $C$ is a convex subset of $\mathscr{R}(M)$, the right cosets of $M$ in $G$, properly containing $M$ so that $C$ is either a maximal Dedekind complete subchain or is an Ohkuma chain. Then $M$ is regular and $P=\{g \in G: M+g \in C\}$ is the cover of $M$. 
Proof. It is well known that the $o$-blocks containing $M$ of the action of $G$ upon $\mathscr{R}(M)$ correspond to the convex $l$-subgroups of $G$ containing $M$; thus $P$ is a convex $l$-subgroup.

Now suppose there exists a convex $l$-subgroup $H$ such that $M \subset H \subset P$.

Suppose that $C$ is Dedekind complete. Since $H \subset P$, there exists $0<a \in$ $P \backslash H$ and so $M+a>M+h$ for any $h \in H$. So $M+g=\bigvee\{M+h: h \in H\}$ exists in $C$. We can assume that $g$ is positive. Now for any $0<h \in H, M+$ $g+h=\left[\bigvee\left\{M+h_{1}: h_{1} \in H\right\}\right]+h=\bigvee\left\{M+h_{1}+h: h_{1} \in H\right\}=M+g$, and so $g+H-g \subseteq M$. But then $M \subset H \subseteq-g+M+g$. Since $M \subset-g+M+g$, $-g+M+g \subset-2 g+M+2 g$. Since $M+g=\bigvee\{M+h: h \in H\}$ and $H \subset-2 g+M+2 g, g \in-2 g+M+2 g$, implying $g \in M$, a contradiction.

If $C$ is an Ohkuma chain, then for any $m \in M, M+m=M$ and so for all $M+g \in C, M+g+m=M+g$, implying that $M$ is normal and $P$ and $P / M$ is then an Ohkuma group and so archimedean.

Note that if $C$ is an Ohkuma chain, then $M$ is normal in its cover $P$. Ohkuma chains are the only chains that guarantee such normality.

Proposition 2.13. Let $T$ be a chain and $M$ be a regular subgroup of an l-group $G$ such that $\mathscr{R}_{\mathscr{M}^{*}}(M)$, the chain of right cosets of $M$ in its cover $M^{*}$, is order isomorphic to $T$. Then $M$ must be normal in $M^{*}$ if and only if $T$ is an Ohkuma chain.

Proof. Suppose that $T$ is not an Ohkuma chain. Since $M^{*}$ acts transitively on $\mathscr{R}_{\mathscr{M}^{*}}(M), \mathscr{A}(T)$ acts transitively on $T$. So there exist $a, b \in T$ and an order automorphism $\tau$ of $T$ so that $\tau(a)=a$ but $\tau(b) \neq b$. There exists an order automorphism $\sigma$ of $T$ so that $\sigma(a)=b$. Then $\left(\sigma^{-1} \circ \tau \circ \sigma\right)(a) \neq a$. So the stabilizer $S_{a}$ is a maximal convex $l$-subgroup of $\mathscr{A}(T)$ that is not normal, but $\mathscr{R}_{\mathscr{A}(T)}\left(S_{a}\right)$ is order isomorphic to $T$.

Proposition 2.14. Let $(G, \leq,+)$ be an o-group and $H$ be a convex subset of $G$ containing 0 that is either a maximal Dedekind complete chain or an Ohkuma chain. Then $H$ is the minimal convex subgroup of $G$, and is invariant under every order-preserving permutation that preserves 0 .

Proof. $H$ is clearly the covering convex subgroup of $(0)$ in $G$ by Corollary 2.12. The rest follows from the fact that for any order-preserving permutation $\tau$ of $G$, either $H \subseteq \tau(H)$ or $\tau(H) \subseteq H$.

Theorem 2.15. Let $(G, \leq,+)$ be an l-group and $H$ be a convex l-subgroup invariant under all lattice automorphisms $\tau$ of $G$ that preserve 0 . Then for any compatible group operation $\oplus$,

(a) $H$ is an l-ideal of $(G, \leq, \oplus)$.

(b) For all $g \in H, H+g=H \oplus g$.

(c) $(G,+) / H=(G, \oplus) / H$ as ordered sets.

Proof. For all $g \in H, g+H-g=H$ and so $H$ is an $l$-ideal of $(G, \leq,+)$. Now $H=(g+H) \ominus g$ implies that $H+g=g+H=H \oplus g$ for all $g \in G$. More, if $g \in H$, this shows $H$ to be a convex subsemigroup of $(G, \leq, \oplus)$. Finally, $\ominus-H=H$ shows $H$ to be closed with respect to inverses under $\oplus$ and so $H$ is a convex $l$-subgroup of $(G, \leq, \oplus)$. The rest is now clear.

Lemma 2.16. Let $(G, \leq,+)$ be an o-group such that $\Gamma(G)$ satisfies the descending chain condition and that each component $G^{\gamma} / G_{\gamma}$ is either an Ohkuma group 
or isomorphic to $\mathscr{R}$. Let $(G, \leq, \oplus)$ also be an l-group. Then $(G, \leq, \oplus)$ has exactly the same convex subgroups as $(G, \leq,+)$ and, for all $\gamma \in \Gamma(G)$,

(a) $G_{\gamma}$ is normal in $(G, \leq,+)$ and $G_{\gamma}$ is normal in $(G, \leq, \oplus)$.

(b) For all $g \in G, G_{\gamma}+g=G_{\gamma} \oplus g$.

(c) $(G,+) / G_{\gamma}=(G, \oplus) / G_{\gamma}$ as ordered sets, and

(d) $\left(G^{\gamma}, \oplus\right) / G_{\gamma}$ is the same group as $\left(G^{\gamma},+\right) / G_{\gamma}$ if $G^{\gamma} / G_{\gamma}$ is Ohkuma.

Proof. We will index $\Gamma(G)$ by an ordinal $\lambda$ so that $G_{0}=(0)$. It is clear that in $\Gamma(G), G_{\beta+1}$ covers $G_{\beta}$ and that if $\alpha$ is a limit ordinal $<\lambda, G_{\alpha}=\bigcup_{\beta<\alpha} G_{\beta}$.

Now $G_{0}$ clearly satisfies all conditions. Let $\alpha<\lambda$ be an ordinal and suppose for all ordinals $\beta<\alpha, G_{\beta}$ is a subgroup of $(G, \leq, \oplus)$ satisfying (a), (b), (c), and (d). If $\alpha=\beta+1$, then by passing to the factor group $G / G_{\beta}$, we can assume that $\alpha=1$.

But $G_{1}$ is Ohkuma or real; by Proposition 2.14, $\tau\left(G_{1}\right)=G_{1}$ for any order automorphism $\tau$ that preserves 0 and so by Theorem $2.15, G_{1}$ satisfies (a), (b), (c), and (d). So for every successor ordinal $\alpha<\lambda$, the theorem is true.

If $\alpha$ is a limit ordinal, then $G_{\alpha}=\bigcup_{\beta<\alpha} G_{\beta}$, each of which is a normal convex subgroup of $(G, \leq, \oplus)$ and so $G_{\alpha}$ is normal in $(G, \leq, \oplus)$. Also, $G_{\alpha} \oplus x=\bigcup_{\beta<\alpha}\left(G_{\beta} \oplus x\right)=\bigcup_{\beta<\alpha}\left(G_{\beta}+x\right)=G_{\alpha}+x$.

Theorem 2.17. Let $(G, \leq,+)$ be a special-valued l-group with $\Delta$ the minimal plenary subset of special values of $\Gamma(G)$. Suppose $\Delta$ satisfies the descending chain condition and that for all $\delta \in \Delta, G^{\delta} / G_{\delta}$ is either Ohkuma or real. Then for any other addition $\oplus$ on $(G, \leq),(G, \leq, \oplus)$ has exactly the same special values and covers of special values as $(G, \leq,+)$.

Proof. As mentioned above, special elements are determined by the lattice and the identity. Let $s$ be special in $(G, \leq,+)$ with value $G_{\delta} . G_{\delta}$ then equals $I_{s} \boxplus s^{\prime}$, where $I_{s}=\{g \in G: n|g|<|s|$ for all integers $n\}$ [1].

Now $s^{\prime \prime}$ is a polar in $(G, \leq, \oplus)$ as well and lex $s^{\prime \prime}$ is the prime subgroup of $s^{\prime \prime}$ in both $(G, \leq,+)$ and $(G, \leq, \oplus) . s^{\prime \prime}$ is also special-valued; the special components of $s^{\prime \prime}$ remain Ohkuma or real; and the special values of $s^{\prime \prime}$ still satisfy the descending chain condition.

So in $s^{\prime \prime} /\left(\right.$ lex $\left.s^{\prime \prime}\right)$, we satisfy the hypotheses of Lemma 2.16 and thus $\left(s^{\prime \prime}, \oplus\right) /\left(\right.$ lex $\left.s^{\prime \prime}\right)$ has exactly the same set of convex $o$-subgroups as does $\left(s^{\prime \prime},+\right) /\left(\right.$ lex $\left.s^{\prime \prime}\right)$. Thus two special elements are $a$-equivalent in $(G, \leq,+)$ if and only if they are a-equivalent in $(G, \leq, \oplus)$ and so each special value $G_{\gamma}$ in $(G, \leq,+)$ remains a special value of exactly the same special elements in $(G, \leq, \oplus)$.

We cannot do away with the hypothesis that $\Delta$ satisfies the descending chain condition; for let $\Sigma(\mathscr{Z}, \mathscr{Z})$ denote those elements of $\mathscr{V}(\mathscr{Z}, \mathscr{Z})$ that have finite support. Then the underlying chain is a countable unbounded chain dense in itself and so is isomorphic to $(\mathscr{Q}, \leq)$.

\section{LATTICE-ORDERED GROUPS WITH UNIQUE ADDITION}

In the preceding section, we established that many subgroups, subgroup structures, and properties of lattice-ordered groups are preserved under any compatible group operation. We are now in a position to apply these results and prove that several important classes of $l$-groups have unique addition. 
Theorem 3.1. For an o-group $G$, the following are equivalent:

(a) $G$ has unique addition.

(b) An order-preserving permutation of $(G, \leq)$ that fixes 0 must be the identity.

(c) The only order-preserving permutations of $(G, \leq)$ are the group translations.

(d) $G$ is an Ohkuma group.

Proof. Clearly (b) $\Rightarrow(\mathrm{c}) \Rightarrow(\mathrm{d}) \Rightarrow(\mathrm{a})$.

For $(\mathrm{a}) \Rightarrow(\mathrm{b})$, let $\tau$ be an order-preserving permutation of $(G, \leq)$ such that $\tau(0)=0$; define $a \oplus b$ to be $\tau^{-1}[\tau(a)+\tau(b)]$ for all $a, b \in G$. Then $+=\oplus$ by hypothesis and so $\tau$ is an $o$-automorphism of $(G, \leq,+)$. Suppose by way of contradiction that $\tau$ is not the identity. Define

$$
\beta(g)= \begin{cases}\tau(g), & \text { if } g>0 ; \\ g, & \text { if } g \leq 0 .\end{cases}
$$

Then $\beta$ is an order automorphism of $G$ with $\beta(0)=0$ but is not an $o$ automorphism.

For an arbitrary $l$-group with unique addition, certain parts of the above theorem are also true.

Proposition 3.2. For an l-group $(G, \leq,+)$ with unique addition, every lattice automorphism of $(G, \leq)$ preserving 0 is a group automorphism and the only p-permutation of $(G, \leq)$ is the identity.

Proof. If $\tau$ is a $p$-permutation of $(G, \leq)$, we can define $\sigma: g \rightarrow \tau\left(g^{+}\right)-g^{-} . \sigma$ is then easily seen to be a lattice automorphism of $(G, \leq)$ preserving 0 and thus is a group automorphism. But then $\tau\left(g^{-}\right)-g^{+}=\sigma(-g)=-\sigma(g)=g^{-}-\tau\left(g^{+}\right)$, implying $g^{-}=\tau\left(g^{-}\right)$and $g^{+}=\tau\left(g^{+}\right)$. So $\tau$ is the identity on $G^{+}$and a similar proof shows $\tau$ to be the identity on $G^{-}$. Since a lattice automorphism preserving 0 is determined by its actions on the positive and negative cones, $\tau$ is the identity.

We suspect but cannot prove that if $(G, \leq)$ has no nontrivial $p$-permutations, then $(G, \leq)$ has unique addition. We can show this to be true if $(G, \leq)$ admits an archimedean addition.

Proposition 3.3. If $(G, \leq,+)$ is archimedean and has no nontrivial p-permutations, then $(G, \leq)$ has unique addition.

Proof. Suppose $\oplus$ is another addition for $(G, \leq)$. Since $(G, \leq,+)$ is archimedean, then for any $0<g \in G$ or any $0>g \in G$, the map $x \rightarrow-g+(x \oplus g)$ is a $p$-permutation and so $x+g=x \oplus g$ for all $x \in G$. Also, for all $g \in G$, $-g=\ominus g$ since the map $g \rightarrow-\ominus g$ is a $p$-permutation. So for any $x, g \in G$, $x+g=\left(x+g^{+}\right)+-g^{-}=\left(x \oplus g^{+}\right) \oplus-g^{-}=\left(x \oplus g^{+}\right) \oplus \ominus g^{-}=x \oplus g$.

The following theorem is central to most of our results of this section.

Theorem 3.4. Suppose that $G$ is an l-group that satisfies

There exists a set $\mathscr{M}$ of convex l-subgroups of $G$ such that $\bigcap \mathscr{M}=(0)$; that for each $M \in \mathscr{M}, \mathscr{R}(M)$ is an Ohkuma

(*) chain; and that each $M \in \mathscr{M}$ contains a minimal prime subgroup $N$ such that $G / N$ has Ohkuma or real components and $\Gamma(G / N)$ satisfies the descending chain condition.

Then $G$ has unique addition. 
Proof. By Proposition 2.13, for any $M \in \mathscr{M}, M$ is normal in $G$ and $G / M$ is an Ohkuma group. Since $\bigcap \mathscr{M}=(0), G$ is archimedean. So if $\oplus$ is any other group operation on $(G, \leq)$, then for any minimal prime subgroup $N$ of $G$, $N$ is normal in $(G, \oplus)$ by Theorem 2.9(a), and for all $x \in G, N+x=N \oplus x$.

Now for $M \in \mathscr{M}$, let $N$ be the minimal prime subgroup contained in $M$ so that $G / N$ has Ohkuma or real components and $\Gamma(G / N)$ satisfies the descending chain condition. Since for all $x \in G, N+x=N \oplus x,(G, \leq,+) / N=$ $(G, \leq, \oplus) / N$ as ordered sets. Applying Lemma 2.16 to $(G, \leq) / N$ shows that $M / N$ is a maximal convex subgroup normal in $(G, \oplus) / N$ and so $M$ is a maximal convex $l$-subgroup normal in $(G, \oplus)$. Also by Lemma $2.16, M+x=$ $M \oplus x$ for all $x \in G$.

Suppose now there exist $a, b \in G$ such that $a+b \neq a \oplus b$. Then there exists $M \in \mathscr{M}$ such that $M+a+b \neq M+(a \oplus b)$. But $M+a+b=$ $(M+a)+(M+b)=(M \oplus a) \oplus(M \oplus b)$ (since $G / M$ has unique addition) $=M+a \oplus b$, a contradiction.

We get several corollaries from this theorem.

Corollary 3.5. If $G$ is a subdirect product of Ohkuma groups, if $\Gamma(G)$ satisfies the descending chain condition, and if each component $G^{\gamma} / G_{\gamma}$ is Ohkuma or real, then $G$ satisfies (*) and hence has a unique addition.

Corollary 3.6. If $G$ is a hyperarchimedean subdirect sum of Ohkuma groups, then $G$ satisfies $(*)$ and so has a unique addition.

Corollary 3.7. If $G$ is a finitely generated archimedean l-group and each component is cyclic, then $G$ has a unique addition.

Proof. If $N$ is a minimal prime subgroup, then $G / N$ is a totally ordered finitely generated abelian group. Thus $\Gamma(G / N)$ is finite and each component is cyclic. Since $G$ is archimedean, finitely generated, and all components are cyclic, $G$ is a subdirect product of integers.

Corollary 3.8. A completely distributive archimedean l-group $G$ has unique addition if and only if $\sum_{\Lambda} K_{\Lambda} \subseteq G \subseteq \prod_{\Lambda} K_{\lambda}$, where each $K_{\lambda}$ is an Ohkuma group.

Proof. If $G$ is a completely distributive archimedean $l$-group, then without loss of generality, $\sum_{\Lambda} A_{\lambda} \subseteq G \subseteq \prod_{\Lambda} A_{\lambda}$, where each $A_{\lambda}$ is an archimedean 0 -group. In particular, $G=A_{\lambda} \boxplus A_{\lambda}^{\prime}$ for all $\lambda$. Now if some $A_{\lambda}$ is not an Ohkuma group, then it admits two additions as an $O$-group and hence $G$ has two additions.

Conversely, suppose $\sum_{\Lambda} K_{\lambda} \subseteq G \subseteq \prod_{\Lambda} K_{\lambda}$, where each $K_{\lambda}$ is an Ohkuma group. For all $\lambda \in \Lambda$, let $G_{\lambda}=\left\{g \in G: g_{\lambda}=0\right\}$. Then $G_{\lambda}$ is a maximal and a minimal prime subgroup of $G$ and $G / G_{\lambda} \cong K_{\lambda}$. So $G$ satisfies (*).

At this time, we know of no subdirect product of Ohkuma groups that do not have unique additions. The following characterizes those that do.

Proposition 3.9. The following are equivalent for any l-group $G$ that is a subdirect product of Ohkuma groups:

(a) $G$ has unique addition.

(b) There exists a set $\mathscr{M}$ of maximal prime subgroups such that $\cap \mathscr{M}=(0)$ and for all $M \in \mathscr{M}, G / M$ is an Ohkuma group and $\tau(M)=M$ for every p-permutation $\tau$ of $(G, \leq)$. 
(c) Each maximal prime subgroup of $G$ is invariant under all p-permutations of the lattice $(G, \leq)$.

(d) The identity is the only p-permutation of $(G, \leq)$.

Proof. We have shown (a) $\Rightarrow$ (d) and clearly (d) $\Rightarrow($ c $) \Rightarrow($ b). For (b) $\Rightarrow$ (a), let $x \in G$. Because $G$ can be $l$-embedded into $\prod_{M \in \mathscr{M}} G / M, G$ is archimedean and thus for all $g \in G$, the map $x \rightarrow-g+(x \oplus g)$ is a $p$ permutation. So for all $g \in G$ and all $M \in \mathscr{M}, M+g=M \oplus g$, and thus $G$ has a unique addition.

We now look at the class $\mathscr{U}^{*}$ of $l$-groups that satisfy $(*)$ of Theorem 3.4 .

Proposition 3.10. If $(G, \leq,+) \in \mathscr{U}^{*}$, so does every l-ideal of $(G, \leq,+)$.

Proof. Let $L$ be an $l$-ideal of $G$. Since the map $P \rightarrow P \cap L$ is an orderpreserving bijection from the primes of $G$ not containing $L$ onto the primes of $L$, this is clear.

Proposition 3.11. If $\left\{A_{\lambda}\right\}_{\Lambda}$ is a set of l-groups where each $A_{\lambda}$ is an element of $\mathscr{U}^{*}$, and $G$ is an l-subgroup of $\prod_{\Lambda} A_{\lambda}$ containing $\sum_{\Lambda} A_{\lambda}$, then $G \in \mathscr{U}^{*}$.

Proof. If $P$ is a prime of $A_{\lambda}$, then $Q=P \boxplus\left[\prod_{\lambda \neq \mu \in \Lambda} A_{\mu}\right]$ is a prime of $\prod_{\Lambda} A_{\lambda}$ and so $Q \cap G$ is a prime of $G$.

Proposition 3.12. $\mathscr{U}^{*}$ is not closed with respect to l-subgroups.

Proof. There exists an Ohkuma group $K$ that contains a nontrivial divisible subgroup $S$ [16]. By Theorem 2.1, $S$ is not an Ohkuma group and so does not satisfy $(*)$.

We now show that free abelian $l$-groups satisfy $(*)$ and this will at last prove Theorem A.

Theorem 3.13. Each free abelian l-group $G$ satisfies $(*)$. Thus each l-ideal of a free abelian l-group has unique addition.

Proof. Let $S$ be a free set of generators for $G$. Then each $0 \neq g \in G$ has a representation $g=\bigvee_{I} \bigwedge_{J} g_{i j}$, where each $g_{i j}$ is in the subgroup [S] generated by $S$ and both $I$ and $J$ are finite. Since $G$ is a subdirect product of integers [26], there exists a maximal prime $M$ such that $g \notin M$ and $G / M$ is cyclic.

Let $s_{1}, s_{2}, \ldots, s_{n}$ be the generators in $S$ needed for this representation of $g$. Then each $g_{i j}$ is in the subgroup $\left[s_{1}, s_{2}, \ldots, s_{n}\right]$ and without loss of generality, we may assume $M+s_{i} \neq M$ for $i=1,2, \ldots, k$ and that $M+s_{i}=M$ for $i=k+1, k+2, \ldots, n$. Thus we have an $l$-homomorphism $\pi$ of $G$ onto $\mathscr{Z}$ such that $g \pi \neq 0, s_{i} \pi \neq 0$ for $1 \leq i \leq k$, and $s_{i} \pi=0$ for $k+1 \leq i \leq n$.

Let $\left\{s_{\lambda}\right\}_{\Lambda}=S \backslash\left\{s_{1}, \ldots, s_{k}\right\}$ with $\Lambda$ being well ordered. Let $H$ be the

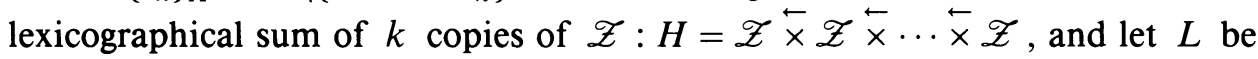
the lexicographical sum $L=\mathscr{V}(\Lambda, \mathscr{Z}) \overleftarrow{\times} H$. 
We define a map $\sigma$ of $S$ into $L$ by

$$
\begin{gathered}
s_{1} \sigma=\left(0,0, \ldots, 0, s_{1} \pi\right) \in H \\
s_{2} \sigma=\left(0,0, \ldots, 0,1, s_{2} \pi\right) \in H \\
\vdots \\
s_{k} \sigma=\left(1,0, \ldots, 0, s_{k} \pi\right) \in H
\end{gathered}
$$

and for $\lambda \in \Lambda, s_{\lambda} \sigma$ is the characteristic function of $\{\lambda\}$ in $\mathscr{V}(\Lambda, \mathscr{Z})$.

Since $G$ is free, $\sigma$ lifts to an $l$-homomorphism $\bar{\sigma}$ of $(G, \leq,+)$ into $L$ with kernel $\operatorname{Ker}(\bar{\sigma})$, and clearly $\bar{\sigma}$ is injective on the subgroup $[S]$. Note $\operatorname{Ker}(\bar{\sigma}) \subseteq M$. Thus for any maximal convex $l$-subgroup $M$ such that $G / M$ is cyclic, there exists a prime subgroup $N=\operatorname{Ker}(\bar{\sigma})$ so that $G / N$ has cyclic components and $\Gamma(G / N)$ satisfies the descending chain condition. So to complete the proof that $G$ satisfies $(*)$, we need only show that $\operatorname{Ker}(\bar{\sigma})$ is a minimal prime subgroup.

Lemma 3.14. Let $S$ be a set of generators of an l-group $G$. If $\pi$ is an $l$ homomorphism of $G$ onto an o-group that induces an isomorphism on the group $[S]$, then $\operatorname{Ker}(\pi)$ is a minimal prime subgroup of $G$.

Proof. Suppose $P$ is a prime subgroup of $G$ contained in $\operatorname{Ker}(\pi)$ and let $0 \neq g \in G$. Then $g=\bigvee_{I} \wedge_{J} g_{i j}$, where $g_{i j} \in S$ and both $I$ and $J$ are finite. Let $\sigma$ be the natural map of $G$ onto the right cosets of $P$. Then $\sigma(g)=P+g=\bigvee_{I} \wedge_{J}\left(P+g_{i j}\right)$.

Now $\Lambda_{J}\left(P+g_{i j}\right)=\min \left\{P+g_{i j}: j \in J\right\}=P+g_{i j^{\prime}}$ for some $j^{\prime} \in J$ and $\bigvee_{I}\left(P+g_{i j^{\prime}}\right)=\max \left\{P+g_{i j^{\prime}}: i \in I\right\}=P+g_{i^{\prime} j^{\prime}}$ for some $i^{\prime} \in I$. Thus $P+g=P+g_{i j}$ for some $i \in I$ and $j \in J$.

Now if $g \in \operatorname{Ker}(\pi)$, then $g_{i j}=p+g \in \operatorname{Ker}(\pi) \cap[S]=(0)$. So $g \in P$ and thus $\operatorname{Ker}(\pi)=P$.

Corollary 3.15. Neither $\mathscr{U}^{*}$ nor the class of l-groups with unique addition is closed with respect to l-homomorphic images.

Recall that an element $0<s$ of an $l$-group is singular if $0<g<s$ implies $g \wedge(s-g)=0$, or that there exists $0<h \in G$ such that $g \wedge h=0$ and $g \vee h=s$. Singular elements are thus recognizable from the lattice and the identity, and if $\tau$ is any lattice automorphism preserving 0 , then $\tau(s)$ is singular if and only if $s$ is singular. Any join of singular elements is again singular and if $s, t$ are singular elements of an $l$-group $G$, then $s \wedge[t-(s \wedge t)]=0$. An archimedean $l$-group is singular if every positive element exceeds a singular element.

A Specker l-group is an l-group that is generated either as an $l$-group or as a group by singular elements. Since each Specker group is a hyperarchimedean subdirect product of integers [8], every Specker group satisfies $(*)$.

Theorem 3.16. A Specker group has unique addition.

Remark. As shown in [8], on a Specker group $G$, there exists a unique multiplication - so that $(G, \leq,+, \cdot)$ is a ring where for any two singular elements $s$ and $t, s \cdot t=s \wedge t$. Moreover, $(G, \leq,+, \cdot)$ is an $f$-ring with zero radical, with each $l$-ideal being a ring ideal, and with each $l$-homomorphism of $G$ being a ring homomorphism. Note that $(G, \leq,+, \cdot)$ is completely determined by the lattice $(G, \leq)$ ! 
Let $S$ be the set of all singular elements of an $l$-group $G$. Then the subgroup $[S]$ is an $l$-ideal of $G$, called the Specker kernel (or radical) of $G$ [8] and [11] and of course is a Specker group.

Theorem 3.17. If $(G, \leq,+)$ and $(G, \leq, \oplus)$ are both l-groups and $[S]$ is the Specker kernel of $(G, \leq,+)$, then $[S]$ is also the Specker kernel of $(G, \leq, \oplus)$ and so $+=\oplus$ on $[S]$.

Proof. Let $s$ be singular in $(G, \leq)$ and suppose that for all positive integers $k \leq n, k s$ is the same under both + and $\oplus$. Then $t=(n s \oplus s)-n s$ is singular and is in $s^{\prime \prime}$; so $t \leq s$. Let $u=s-t$. Then $u \wedge t=0$ and $t \vee u=s$. So $0=[\ominus n s+(t+n s)] \wedge[\ominus n s \oplus(u+n s)]=s \wedge[\ominus n s \oplus(u+n s)]$, a contradiction since $u \in s^{\prime \prime}$. Note $n s \oplus s=n s+s$.

Now for any singular element $s, t=-\ominus s$ is singular and in $s^{\prime \prime}$ by Proposition 2.7. So $t \leq s$. But $s=\ominus-t$ implies $s \leq t$. So for any singular element $s,-s=\ominus s$. Arguing as above, but this time for $-s$, all multiples of $-s$ agree in both $(G, \leq,+)$ and $(G, \leq, \oplus)$.

For any $g \in[S]$ in $(G, \leq,+)$, we can find a pairwise disjoint collection of singular elements $\left\{s_{1}, s_{2}, \ldots, s_{k}\right\}$ and integers $\left\{m_{1}, m_{2}, \ldots, m_{k}\right\}$ so that $g=m_{1} s_{1}+\cdots+m_{k} s_{k}$, with $g^{+}=\left(m_{1} \vee 0\right) s_{1}+\cdots+\left(m_{k} \vee 0\right) s_{k}$ and $g^{-}=$ $\left(-m_{1} \vee 0\right) s_{1}+\cdots+\left(-m_{k} \vee 0\right) s_{k}$. So $g^{+}$and $g^{-}$are in the Specker kernel of $(G, \leq, \oplus)$. The reverse inclusion is proved similarly.

Corollary 3.18. Let $(G, \leq,+)$ be an l-group and $S$ be the set of singular elements of $(G, \leq)$. For every lattice automorphism $\tau$ of $(G, \leq)$ preserving 0 , $\tau([S])=[S]$.

Proof. Define for all $g, h \in G, g \oplus h=\tau\left[\tau^{-1}(g)+\tau^{-1}(h)\right]$. Since $\tau$ is now an $l$-isomorphism from $(G, \leq,+)$ onto $(G, \leq, \oplus), \tau([S])$ is the Specker kernel of $(G, \leq, \oplus)$. Bug $[S]$ is the Specker kernel of $(G, \leq,+)$ and so $\tau([S])=[S]$.

We can now prove Theorem $\mathrm{B}$.

Theorem 3.19. If $(G, \leq,+)$ is a singular archimedean l-group, then $G$ has a unique addition.

Proof. Let $S$ be the set of singular elements of $G$; then $G=S^{\prime \prime}$. For every $p$-permutation $\tau$ of $G$ and every $s \in S, t=\tau(s)$ is in $s^{\prime \prime}$ and so $t \leq s$. Likewise, $s \leq t$ and so $\tau$ is the identity on $S$. By a proof similar to the first part of Theorem 3.17, $\tau(n s)=n s$ for any integer $n \geq 0$.

Define $\sigma(g)$ to be $-[\tau(-g)]$. By Proposition 2.6, $\sigma$ is then a $p$-permutation and so for every $s \in S,-s=\tau(-s)$. So for any integer $n, \tau(n s)=n s$ and thus for all $g \in[S], \tau(g)=g$.

But $[S]$ is a dense convex $l$-subgroup of $(G, \leq,+)$ and so every $0 \leq x \in G$ is the join of all $0 \leq g \in[S]$ such that $g \leq x$. So for all $0 \leq g \in G$, $\tau(g)=g$. This is easily established as well for all $0>g \in G$ and since lattice automorphisms of $(G, \leq)$ that preserve 0 are determined by their actions on the positive and negative cones, $\tau(g)=g$ for all $g \in G$. By Proposition 3.3, $G$ has unique addition.

In [11], there was given an example of a singular archimedean $l$-group $G$ such that $G$ cannot be embedded into a product of real groups. By Theorem 
$3.19, G$ has unique addition. Our previous examples of $l$-groups with unique addition were all subdirect products of reals.

Corollary 3.20. If $G$ is a Specker group, then the lateral completion $G^{L}$ and the Dedekind completion $G^{\wedge}$ of $G$ have unique additions.

Every example so far of an $l$-group with unique addition has been archimedean. It is easy to see that any $l$-group with unique addition must be abelian, for if $(G, \leq,+)$ is any $l$-group and $g, h \in G$, defining $g \oplus h$ to be $h+g$ makes $(G, \leq, \oplus)$ into an $l$-group. We suspect that every $l$-group with unique addition must be archimedean. The next proposition is the best we have been able to prove on this line. The following proof is not ours but is due to a referee.

Proposition 3.21. If $(G, \leq,+)$ is completely distributive and has unique addition, then $(G, \leq,+)$ is archimedean.

Proof. First note that $G$ must be abelian.

Let $0<a \in G$. Since $G$ is completely distributive, there exists $g \in G$ such that $g>0$ and for any set $A$ of positive elements of $G$ satisfying $a=\bigvee A$, there exists $b \in A$ such that $g \leq b$. Define $H$ to be $G(a)=\{x \in G:|x| \leq n a$ for some integer $n\}$; let $K=H+g^{\prime}$. Let $V$ be a value of $g$ and $0 \leq v \in V$.

Since $G$ is abelian, $a=a \wedge v+(a-a \wedge v) \leq 2(a \wedge v) \vee 2(a-a \wedge v)$. Since $g \notin V, g \not \leq 2(a \wedge v)$, and so $g \leq 2(a-a \wedge v)$. Thus $v-a \wedge v \in g^{\prime}$. But $a \wedge v \in V$, and so $v \in K$.

Now suppose that $x \in K, y \notin K$, and $x<y$. Clearly $x-g \leq y$. If $g \wedge(x+g-y)^{+} \neq 0$, there is a value of $g$ which contains $(x+g-y)^{-}=$ $(y-(x+g))^{+}$, and hence $(y-(x+g))^{+} \in K$. Since $(x+g) \wedge y \in K$, this gives the contradiction that $y \in K$. Thus $g \wedge(x+g-y)^{+}=0$, and since $(x+g-y)^{+} \leq g$, we have $(x+g-y)^{-}=0$, and $x+g \leq y$. It follows that the map $\tau: G \rightarrow G$ defined by

$$
\tau(u)= \begin{cases}u+a, & u \notin K ; \\ u, & u \in K .\end{cases}
$$

is an order automorphism of $G$. Since addition is unique, $\tau$ is an $l$-automorphism of $G$. Thus for all $u \in G \backslash K, 2 u=(u+a+u+a)-a=2 u+a$, which is impossible since $a \neq 0$. Thus $K=G$. In particular, for any $B \in G^{+}$, there exists an integer $n$ such that $b-b \wedge n a \in g^{\prime}$ and $((n+1) a-b)^{+} \geq$ $(a-(b-b \wedge n a))^{+} \geq g$; so $(n+1) a \leq b$ is impossible. So $G$ is archimedean.

\section{4. l-GROUPS WITH ESSENTIALLY ONLY ONE ADDITION}

In the preceding section, we generalized two properties of the integersthat they form a uniquely transitive chain and that 1 is a singular element-to obtain the class $\mathscr{U}^{*}$ of $l$-groups satisfying $(*)$ of Theorem 3.4 and to show that singular archimedean $l$-groups, respectively, have unique additions. In this section, we generalize the real number chain.

As before, let $(\mathscr{R}, \leq,+)$ be the group of real numbers with the usual order and addition. If $(\mathscr{R}, \leq, \oplus)$ is an $o$-group, then since $(\mathscr{R}, \leq)$ is Dedekind complete and has no singular elements, $(\mathscr{R}, \leq, \oplus)$ is $o$-isomorphic to $(\mathscr{R}, \leq$, $+)$ but + need not equal $\oplus$. Thus the ordered set $(\mathscr{R}, \leq)$ has essentially only one addition + such that $(\mathscr{R}, \leq,+)$ is an $o$-group. 
Let $(G, \leq,+)$ be a subgroup of $(\mathscr{R}, \leq,+)$ and let $\oplus$ be an addition on $(G, \leq)$ such that $(G, \leq, \oplus)$ is archimedean. Then $\oplus$ can be extended to an addition on $(\mathscr{R}, \leq)$ so that $(\mathscr{R}, \leq, \oplus)$ is an $o$-group. Thus each new archimedean addition of $(G, \leq)$ is determined by an order automorphism $\tau$ of $(\mathscr{R}, \leq)$ where $\tau(G)$ is a subgroup of $(\mathscr{R}, \leq,+)$.

However, we cannot assume that if $(G, \leq, \oplus)$ is archimedean, then $(G, \leq$, $\oplus)$ is $l$-isomorphic to $(G, \leq,+)$. For $(\mathscr{Q}, \leq,+)$ is as a chain order-isomorphic to $(\mathscr{Q}+\mathscr{Q} \sqrt{2}, \leq,+)$ with the usual order and addition. So we can place new archimedean additions on $(\mathscr{Q}, \leq)$ not isomorphic to the usual addition.

To generalize these ideas, let $X$ be the Stone space associated with the complete Boolean algebra of polars of an $l$-group $G . X$ is then a compact Hausdorff extremally disconnected space. Let $\mathscr{D}(X)$ be the set of all functions $f$ from $X$ into the extended real numbers $[-\infty,+\infty]$ so that $f$ is a continuous real-valued function on a dense open subset of $X$. Then $\mathscr{D}(X)$ is an archimedean $f$-ring under pointwise order, addition, and multiplication. Bernau [2] proved that if $G$ is an archimedean $l$-group, there exists an $l$ embedding $\pi$ of $G$ into $\mathscr{D}(X)$ such that $\mathscr{D}(X)$ is an essential extension of $G$ : each nonzero $l$-ideal of $\mathscr{D}(X)$ has nonzero intersection with $G \pi$. In fact, $\mathscr{D}(X)$ is the unique archimedean essential closure of $G$ [7]. We will identify $G$ with $G \pi$. Then $G \subseteq \mathscr{D}(X) \cong G^{d \wedge L}=G^{d L \wedge}$, where $G^{d}$ is the divisible hull of $G, G^{L}$ is the lateral completion of $G$, and $G^{\wedge}$ is the Dedekind-MacNeille completion of $G$.

Theorem 4.1. For any Stone space $X, \mathscr{D}(X)$ has essentially only one addition.

Proof. Let + be the usual addition on $\mathscr{D}(X)$ and let $\oplus$ be another addition on $(\mathscr{D}(X), \leq)$. Since $(\mathscr{D}(X), \leq)$ is complete, $(\mathscr{D}(X), \leq, \oplus)$ is archimedean. Because the polars of $(\mathscr{D}(X), \leq, \oplus)$ are those of $(\mathscr{D}(X), \leq,+),(\mathscr{D}(X), \leq$, $\oplus)$ can be $l$-embedded into $(\mathscr{D}(X), \leq,+)$ as a dense $l$-subgroup, and $(\mathscr{D}(X)$, $\leq,+) \cong(\mathscr{D}(X), \leq, \oplus)^{\wedge \mathscr{L}}$. But $(\mathscr{D}(X), \leq)$ is complete, laterally complete, and has no singular elements; so $(\mathscr{D}(X), \leq, \oplus)$ is divisible, laterally complete, and complete.

So every two additions on $(\mathscr{D}(X), \leq)$ are connected by a lattice automorphism of $(\mathscr{D}(X), \leq)$. Note that we have also proved Theorem $\mathrm{C}$.

Theorem 4.2. Let $(G, \leq,+)$ be an archimedean l-group and $(\mathscr{D}(X), \leq,+)$ be the essential closure of $(G, \leq,+)$. Let $\tau$ be a lattice automorphism of $(\mathscr{D}(X), \leq)$ preserving 0 and define $a \oplus b$ to be $\tau^{-1}[\tau(a)+\tau(b)]$ for all $a, b$ in $\mathscr{D}(X)$.

Then $\oplus$ defines a new archimedean addition for $(G, \leq)$ if and only if $\tau(G)$ is an l-subgroup of $(\mathscr{D}(X), \leq,+)$ and ever archimedean addition for $(G, \leq)$ is obtained in this way.

Proof. Let $\oplus$ be a new archimedean addition for $(G, \leq)$. Since the polars of $(G, \leq, \oplus)$ are those of $(G, \leq,+)$, the Stone space of $(G, \leq, \oplus)$ is homeomorphic to that of $(G, \leq,+)$ and so $(H, \leq, \oplus)$, the essential closure of $(G, \leq, \oplus)$, is $l$-isomorphic to $(\mathscr{D}(X), \leq,+)$ by way of some $l$-isomorphism $\pi$. Note that $\pi$ restricts to a lattice isomorphism $\tau$ of the subset $(G, \leq)$ of $(\mathscr{D}(X), \leq)$ onto another subset $(\tau(G), \leq)$ of $(\mathscr{D}(X), \leq)$. We will now show that $\tau$ can be extended to a lattice automorphism of $(\mathscr{D}(X), \leq)$. 
To start, the Dedekind completions of both $(G, \leq)$ and $(\tau(G), \leq)$ are lattice constructions within $(\mathscr{D}(X), \leq)$ and so $\tau$ can clearly be extended to a lattice isomorphism from $(G, \leq)^{\wedge}$ onto $(\tau(G), \leq)^{\wedge}$. So we can assume that $(G, \leq)$ is complete.

Then $(G, \leq)=G_{v} \boxplus G_{s}$, where $G_{v}$ is a complete vector lattice (under + or $\oplus)$ and $G_{s}$ is a complete singular archimedean l-group [11]. Similarly, $(\tau(G), \leq)^{\wedge}$ decomposes as $\tau(G)_{v} \boxplus \tau(G)_{s}$. Since $\tau$ must map singular elements to singular elements, clearly $\tau\left(G_{v}\right)=\tau(G)_{v}$ and $\tau\left(G_{s}\right)=\tau(G)_{s}$.

Now $G_{s}$ is a singular archimedean $l$-group and so has unique addition; thus $+=\oplus$ on $G_{s}$ and then $\tau$ lifts to an $l$-isomorphism between $\left(G_{s}, \leq, \oplus\right)^{d}$ and $\left(\tau\left(G_{s}\right), \leq,+\right)^{d}=\left(\tau(G)_{s}, \leq,+\right)^{d}$; this gives us a lattice isomorphism again inside $(\mathscr{D}(X), \leq)$ of $(G, \leq, \oplus)^{d}$ onto $(\tau(G), \leq,+)^{d}$ which again extends to a lattice isomorphism from $(G, \leq, \oplus)^{d}$ onto $(\tau(G), \leq,+)^{d}$.

Now $\tau(G)$ is large in $(\mathscr{D}(X), \leq,+)$ because $(G, \leq, \oplus)$ is large in $(H, \leq$, $\oplus)$. Jakubik [20] has shown that $(\tau(G), \leq)^{d}$ actually defines $(\mathscr{D}(X), \leq)$ as a lattice and so $\tau$ can be extended to $(\mathscr{D}(X), \leq)$. Note that defining $*$ on $(\mathscr{D}(X), \leq)$ by $g * h=\tau^{-1}[\tau(g)+\tau(h)]$ yields that $*=\oplus$ on $(G, \leq)$ and so we can extend $\oplus$ to $(\mathscr{D}(X), \leq)$.

The rest of the proof is obvious.

When $G$ is a completely distributive archimedean $l$-group, we can make a useful restriction on the action of the lattice automorphism $\tau$. For in this case, we can assume that $\sum_{\Lambda} A_{\lambda} \subseteq G \subseteq \prod_{\Lambda} A_{\lambda}$, where each $A_{\lambda}$ is an archimedean $o$ group, and so $(\mathscr{D}(X), \leq,+)$ is $l$-isomorphic to $\prod_{\Lambda} \mathscr{R}_{\lambda}$. Now if $\tau$ is a lattice isomorphism of $\prod_{\Lambda} \mathscr{R}_{\lambda}$, then for all $\lambda, \tau\left(\mathscr{R}_{\lambda}\right)=\mathscr{R}_{\mu}$ for some $\mu \in \Lambda$. Thus $\tau$ determines a permutation $\pi$ of $\Lambda: \lambda \rightarrow \mu$. $\pi$ in its turn defines a lattice automorphism $\sigma$ of $\prod_{\Lambda} \mathscr{R}_{\lambda}$ by $[\sigma(x)]_{\lambda}=x_{\pi^{-1}(\lambda)}$. Now for all $\lambda, \sigma^{-1} \circ \tau$ preserves each $\tau$ and so induces a lattice automorphism $\tau_{\lambda}$ of $\mathscr{R}_{\lambda}$. Let $\alpha$ denote $\sigma^{-1} \circ \tau$; then $[\alpha(x)]_{\lambda}=\tau_{\lambda}\left(x_{\lambda}\right)$. Defining $\oplus$ by $g \oplus h=\tau^{-1}[\tau(g)+\tau(h)]$, we see that $g \oplus h=\alpha^{-1}[\alpha(g)+\alpha(h)]$. Thus we can assume that $\tau$ preserves each stalk $\mathscr{R}_{\lambda}$. This generalizes to the following proposition.

Proposition 4.3. For a Stone space $X$, the group of all lattice automorphisms of $(\mathscr{D}(X), \leq)$ that preserve 0 is a splitting extension of the p-permutations by the ring l-automorphisms of $(\mathscr{D}(X), \leq,+, \cdot)$.

Proof. Let $\tau$ be a lattice automorphism of $(\mathscr{D}(X), \leq)$ preserving 0 and let $\oplus$ be the addition defined from $\tau$ in the usual way. Then $\tau$ is an $l$-isomorphism of $(\mathscr{D}(X), \leq,+)$ onto $(\mathscr{D}(X), \leq, \oplus)$ and so for every minimal prime subgroup $M$ of $(\mathscr{D}(X), \leq,+), \tau(M)$ is also a minimal prime subgroup under both + and $\oplus$. Thus $\tau$ induces a permutation $\pi$ on the minimal primes of $(\mathscr{D}(X), \leq)$.

For all $x \in X, D_{x}=\{g \in(\mathscr{D}(X), \leq,+): g(x)=0\}$ is easily seen to be a maximal prime subgroup. Since $\mathscr{D}(X)$ is complete, there exists a unique minimal prime subgroup $M_{x} \subseteq D_{x}$ and $M_{x}=\bigcup\left\{f^{\prime}: 0 \leq f \in \mathscr{D}(X) \backslash D_{x}\right\}$.

Let $M$ be a minimal prime subgroup of $(\mathscr{D}(X), \leq,+)$. Since $M$ is also a ring ideal, $M$ cannot contain any multiplicative units of $(\mathscr{D}(X), \leq,+, \cdot)$ and, since multiplicative units of $\mathscr{D}(X)$ are precisely the weak order units, $M$ contains no weak order units. 
Now suppose that for all $x \in X, M \neq M_{x}$. Choose $0<g_{x} \in M \backslash M_{x}$. Now $\operatorname{supp}\left(g_{x}\right)=\{y \in X: g(y) \neq 0\}$ is open in $X$ and $\bigcup_{x \in X} \operatorname{supp}\left(g_{x}\right)=X$. By the compactness of $X$, there exist $g_{x_{1}}, \ldots, g_{x_{n}}$ such that $X=\bigcup_{1 \leq i \leq n} \operatorname{supp}\left(g_{x_{i}}\right)$. Then $h=g_{x_{1}} \wedge \cdots \wedge g_{x_{n}}$ is in $M$ and is a weak order unit. Thus $M$ must be $M_{x}$ for some $x \in X$.

Thus $\pi$ induces a permutation $\bar{\pi}$ on $X$ that is easily seen to be a homeomorphism of $X$ [5]. $\bar{\pi}$ in its turn defines a ring $l$-automorphism $\sigma$ of $(\mathscr{D}(X), \leq,+, \cdot)$ by $(f \sigma)(x)=f(\bar{\pi}(x))$.

Now for any $g \in M_{x}, \tau(g) \in M_{\bar{\pi}(x)}$ implies that there exists $0<f \in \mathscr{D}(X)$ such that $f(\bar{\pi}(x)) \neq 0$ but $\tau(g) \wedge f=0$. Then $[\tau(g)] \sigma \wedge f \sigma=0$ and $(f \sigma)(x)=$ $f(\bar{\pi}(x)) \neq 0$, giving that $\tau(g) \in M_{x}$. Therefore, $\rho=\sigma \circ \tau$ is a $p$-permutation of $(\mathscr{D}(X), \leq)$ and $\tau=\sigma^{-1} \circ \rho$.

The $p$-permutations clearly form a normal subgroup of the group of lattice automorphisms that preserve 0 . So suppose that $\tau=\alpha \circ \beta$, where $\alpha$ is a ring $l$-automorphism and $\beta$ is a $p$-permutation. Then $\sigma \circ \alpha$ is a $p$-permutation and a ring $l$-automorphism; hence $\sigma \circ \alpha$ is the identity.

Remark. The proposition above also generalizes a description of the l-group automorphisms of $\mathscr{D}(X)$ that appears in [5].

Note that if we define an addition $*$ from $\rho$ as before, then $*=\oplus$, and so with regard to new additions on $(\mathscr{D}(X), \leq)$, we need only consider those additions defined by $p$-permutations.

Now for any set $\Lambda, \prod_{\Lambda} \mathscr{R}_{\lambda}$ has essentially only one addition by Theorem 4.1. It is equally clear to see that $\sum_{\Lambda} \mathscr{R}_{\lambda}$ also has essentially only one addition. However, for an l-subgroup $(G, \leq,+)$ of $\prod_{\Lambda} \mathscr{R}_{\lambda}$ containing $\sum_{\Lambda} \mathscr{R}_{\lambda}$, this may not be true. Indeed, in the examples at the end of this section, a new addition on such an $l$-subgroup $(G, \leq,+)$ with a strong order unit may not preserve the strong order unit. In what follows, we investigate when $(G, \leq,+)$ does have essentially only one addition.

Proposition 4.4. Let $g$ be the characteristic function of $\Lambda$ in $\Pi_{\Lambda} \mathscr{R}_{\lambda}$. Then the l-subgroup $G=\sum_{\Lambda} \mathscr{R}_{\lambda}+[g]$ of $\Pi_{\Lambda} \mathscr{R}_{\lambda}$ has essentially only one addition.

Proof. Let $\oplus$ be a new addition for $G$. Then $\oplus$ is given by a set of order automorphisms $\left\{\tau_{\lambda}\right\}_{\lambda \in \Lambda}$ of the $\mathscr{R}_{\lambda}$ 's so that $\tau_{\lambda}(0)=0$ for each $\lambda, \tau\left(\ldots, g_{\lambda}, \ldots\right)=$ $\left(\ldots, \tau_{\lambda}\left(g_{\lambda}\right), \ldots\right)$, and $\oplus$ is defined from $\tau$ in the usual way.

If we follow $\tau$ by the $l$-automorphism $\alpha$ of $\prod_{\Lambda} \mathscr{R}_{\lambda}$ that is multiplication by the element $\left(\ldots, \frac{1}{\tau_{\lambda}(1)}, \ldots\right)$, we have that $\alpha(\tau(g))=g$. Since $\alpha(\tau(G))$ is an l-subgroup of $\prod_{\Lambda} \mathscr{R}_{\lambda}, G \subseteq \alpha(\tau(G))$ and so we can assume both that $G \subseteq \tau(G)$ and that $\tau(g)=g$.

Now $\sum_{\Lambda} \mathscr{R}_{\lambda}$ is invariant under every lattice automorphism $\sigma$ of $(G, \leq)$ and so $\sum_{\Lambda} \mathscr{R}_{\lambda}$ is an $l$-ideal of $(G, \leq, \oplus)$ with

$$
\mathscr{Z} \cong(G, \oplus) /\left(\sum_{\Lambda} \mathscr{R}_{\lambda}\right)=(G,+) /\left(\sum_{\Lambda} \mathscr{R}_{\lambda}\right)
$$

as ordered sets and $(G, \oplus) /\left(\sum_{\Lambda} \mathscr{R}_{\lambda}\right) \cong(\tau(G),+) /\left(\sum_{\Lambda} \mathscr{R}_{\lambda}\right)$ as ordered groups. So there exists $0<x \in \tau(G)$ such that $\sum_{\Lambda} \mathscr{R}_{\lambda}+x$ generates $(\tau(G),+) /\left(\sum_{\Lambda} \mathscr{R}_{\lambda}\right)$. But now $\sum_{\Lambda} \mathscr{R}_{\lambda}+g=\sum_{\Lambda} \mathscr{R}_{\lambda}+n x$ for some integer $n>0$.

Without loss of generality, $x=\left(\ldots, \frac{1}{n}, \ldots\right)$. Let $h=\tau^{-1}(x)$. If $n>1, h$ 
differs from $g$ in every component and so $\sum_{\Lambda} \mathscr{R}_{\lambda}<\sum_{\Lambda} \mathscr{R}_{\lambda}+h<\sum_{\Lambda} \mathscr{R}_{\lambda}+g$ in $\left.(G,+) / \sum_{\Lambda} \mathscr{R}_{\lambda}\right)$, a contradiction.

In contrast to this, $\sum_{\Lambda} \mathscr{R}+\mathscr{Q}[g]$ admits nonisomorphic additions. Let $\alpha$ be an order isomorphism of the chain $(\mathscr{Q}, \leq)$ onto the chain $(\mathscr{Q}+\mathscr{Q} \sqrt{2}, \leq)$ so that $\alpha(0)=0 . \alpha$ can then be lifted to an order automorphism $\bar{\alpha}$ of $\mathscr{R}$. Let $\tau_{\lambda}=\bar{\alpha}$ for all $\lambda$. Then $\tau(G)=\sum_{\Lambda} \mathscr{R}_{\lambda}+(\mathscr{Q}+\mathscr{Q} \sqrt{2}) g$. Defining $\oplus$ in the usual way from $\tau$, we have that $(G, \oplus) /\left(\sum_{\Lambda} \mathscr{R}_{\lambda}\right) \cong(\tau(G),+) /\left(\sum_{\Lambda} \mathscr{R}_{\lambda}\right) \cong \mathscr{Q}+\mathscr{Q} \sqrt{2} \not$ $\mathscr{Q} \cong(G,+) /\left(\sum_{\Lambda} \mathscr{R}_{\lambda}\right)$. But if $(G, \leq,+)$ were $l$-isomorphic to $(G, \leq, \oplus)$ by some map $\sigma, \sigma$ must induce an isomorphism of $(G,+) /\left(\sum_{\Lambda} \mathscr{R}_{\lambda}\right)$ onto $(G, \oplus) /\left(\sum_{\Lambda} \mathscr{R}_{\lambda}\right)$.

At this time, we do not know if $\sum_{\Lambda} \mathscr{R}_{\lambda}+\mathscr{R}[g]$ has essentially only one addition. We can prove the following.

Proposition 4.5. Let $(G, \leq,+)$ be an l-subgroup of $\prod_{\Lambda} \mathscr{R}_{\lambda}$ so that $G /\left(\sum_{\Lambda} \mathscr{R}_{\lambda}\right)$ is l-isomorphic to $\mathscr{R}_{1} \boxplus \mathscr{R}_{2} \boxplus \cdots \boxplus \mathscr{R}_{n}$, where each $\mathscr{R}_{j} \cong \mathscr{R}$. Then $G$ has essentially only one addition as a real vector lattice.

Proof. $G /\left(\sum_{\Lambda} \mathscr{R}_{\lambda}\right)=\left(\sum_{\Lambda} \mathscr{R}_{\lambda}+\mathscr{R} g_{1}\right) \boxplus \cdots \boxplus\left(\sum_{\Lambda} \mathscr{R}_{\lambda}+\mathscr{R} g_{n}\right)$ and we may assume that the $g_{i}$ 's are pairwise disjoint. Furthermore, by taking a suitable l-automorphism of $\prod_{\Lambda} \mathscr{R}_{\lambda}$, we can assume that each $g_{i}$ is the characteristic function of a subset $\Delta_{i}$ of $\Lambda$. Then

$$
G=\left(\sum_{\Delta_{1}} \mathscr{R}+\mathscr{R}\left[g_{1}\right]\right) \boxplus \cdots \boxplus\left(\sum_{\Delta_{n}} \mathscr{R}+\mathscr{R}\left[g_{n}\right]\right) .
$$

So it suffices to show that each $\sum_{\Lambda_{i}} \mathscr{R}+\mathscr{R}\left[g_{i}\right]$ has essentially only one addition.

So let $\oplus$ be another addition on $H=\sum_{\Delta_{i}} \mathscr{R}+\mathscr{R}\left[g_{i}\right]$ so that we get a real vector lattice. We can assume that $\oplus$ is connected to + by some lattice automorphism $\tau=\left(\ldots, \tau_{\lambda}, \ldots\right)$, that $(\tau(H), \leq,+)$ is a vector sublattice of $\sum_{\Delta_{i}} \mathscr{R}$, and that $\tau\left(g_{i}\right)=g_{i}$. So $H \subseteq \tau(H)$.

$(\tau(H), \leq,+) /\left(\sum_{\Delta_{i}} \mathscr{R}_{\delta}\right) \cong \mathscr{R}$, and so for any $0<x \in \tau(H) \backslash\left(\sum_{\Delta_{i}} \mathscr{R}_{\delta}\right)$, there exists an $r \in \mathscr{R}$ such that $g_{i}=r x+t$ for some $t \in \sum_{\Delta_{i}} \mathscr{R}_{\lambda}$. But then $x=\frac{1}{r}\left(g_{i}-t\right) \in H$. So $\tau(H)=H$ and again we are done.

An immediate corollary to the above is that $\sum_{\Lambda} \mathscr{R}_{\lambda}+\mathscr{R}[g]$ has essentially only one addition as a vector lattice.

In this section, completeness of either $\mathscr{R}$ or $\mathscr{D}(X)$ has been the crucial factor in the proofs. Every complete $l$-group $G$ is the cardinal sum of a complete vector lattice $G_{v}$ and a complete singular $l$-group $G_{s}$. Now if $S$ is the set of singular elements of $G$, then $G_{s}=S^{\prime \prime}$ and so $G_{s}$ has unique addition; since $G_{s}$ is a polar of $G$, a new operation can differ from + only on $G_{v}$. Note that since $\left(G_{v}, \leq\right)$ is complete and has no singular elements, then under any addition $\oplus,\left(G_{v}, \leq, \oplus\right)$ must be an archimedean real vector lattice. So to pursue the study of new additions on complete l-groups, we can assume that $(G, \leq,+)$ is a complete real vector lattice and is a dense $l$-ideal of $\mathscr{D}(X)$ for the Stone space of $\mathscr{P}(G)$, the set of polars of $G$.

Proposition 4.6. Suppose that $(G, \leq,+)$ is a complete vector lattice with a strong order unit $u$ and $(G, \leq, \oplus)$ is an l-group. Then $(G, \leq,+) \cong(G, \leq, \oplus)$ if and only if $(G, \leq, \oplus)$ has a strong order unit. 
Proof. One way is clear. So suppose $(G, \leq, \oplus)$ has a strong order unit. Let $X$ be the Stone space of $\mathscr{P}(G)$ for both $(G, \leq,+)$ and $(G, \leq, \oplus)$. Let $\overline{1}$ be the multiplicative identity of $\mathscr{D}(X)$. There exists an $l$-embedding $\alpha$ of $(G, \leq,+)$ into $(\mathscr{D}(X), \leq,+)$ so that $u \alpha=\overline{1}$. Since $(G, \leq,+)$ is a complete vector lattice, $G \alpha$ is an $l$-ideal of $(\mathscr{D}(X), \leq,+)$ and so $G \alpha \cong C(X)$. But $(G, \leq, \oplus)$ has a strong order unit and so there exists an $l$-embedding $\beta$ of $(G, \leq, \oplus)$ onto $C(X)$ as well.

By combining this with earlier results about singular archimedean l-groups, we can drop the hypothesis that $G$ be a vector lattice.

Corollary 4.7. If $(G, \leq,+)$ is a complete l-group with a strong order unit and if $(G, \leq, \oplus)$ is also an l-group with a strong order unit, then $(G, \leq,+) \cong$ $(G, \leq, \oplus)$.

We now build an example of a complete and dense lattice $(G, \leq)$ with one addition + that makes $(G, \leq,+)$ into an $l$-group with a strong order unit and with two nonisomorphic additions $\oplus$ such that $(G, \leq, \oplus)$ does not have a strong order unit.

Let $(G, \leq,+)$ be a complete real vector lattice with a strong order unit. As outlined in the proof of Proposition 4.6, we can assume that $(G, \leq,+)$ is $l$-isomorphic to $C(X)$ for the Stone space $X$ of $\mathscr{P}(G)$.

Now for any other addition $\oplus$, there exists a lattice automorphism $\tau$ of $D=\mathscr{D}(X)$ such that $\tau(0)=0, \tau(G)$ is an $l$-ideal of $D$, and $\tau$ is an $l$ isomorphism of $(G, \leq, \oplus)$ onto $(\tau(G), \leq,+)$. Let $\overline{1}$ be the characteristic function of $X, \overline{2}$ be twice $\overline{1}, \overline{3}$ be three times $\overline{1}$, etc. We can assume that $\tau(\overline{1})=\overline{1}$. Then $C(X)=D(\tau(\overline{1})) \subseteq D(\tau(\overline{2})) \subseteq D(\tau(\overline{3})) \subseteq \cdots$ and $\tau(G)=$ $\bigcup_{n} D(\tau(\bar{n}))$. (Recall that $D(x)$ is the convex $l$-subgroup of $G$ generated by $x$ and so equals $\{g \in D:|g| \leq n|x|$ for some positive integer $n\}$.) If only a finite number of the $D(\tau(\bar{n}))$ are distinct, then $\tau(G)$ has a strong order unit and so $(G, \leq,+) \cong(G, \leq, \oplus)$. Otherwise, $\tau(G)=\bigcup_{n} D(\tau(\bar{n}))$, where, without loss of generality, $D(\tau(\overline{1})) \subset D(\tau(\overline{2})) \subset \cdots$.

Now to the example:

Example 4.8. Let $(G, \leq,+)$ be the bounded real sequences with pointwise order and addition; then $(G, \leq,+)$ is the $l$-ideal of $D=\mathscr{D}(X)=\prod_{1 \leq i<\infty} \mathscr{R}_{i}$ generated by $\overline{1}$. Recall that we can define a lattice automorphism of $D$ by defining its action on each stalk $\mathscr{R}_{i}$. Now on $\mathscr{R}_{i}$, there exists an order automorphism $\tau_{i}$ such that for a positive integer $n, \tau_{i}(n)=(i+1)^{n-1}$. Then

$$
\tau(\overline{1})=\overline{1}, \quad \tau(\overline{2})=(2,3,4,5, \ldots), \quad \tau(\overline{3})=\left(2^{2}, 3^{2}, 4^{2}, \ldots\right) \text {, etc. }
$$

Since $\left.\sum_{i} \mathscr{R} \subseteq(\mathscr{G}, \leq,+), \tau(G)=\bigcup_{1 \leq i} D\left(1^{i}, 2^{i}, 3^{i}, \ldots\right)\right)$. But then $\tau(G)$ has no strong order unit.

Example 4.9. A similar lattice automorphism can now be used to give a second addition on the $l$-group of bounded real sequences, again having no strong order unit, that is not isomorphic to the addition defined in Example 4.8. Let $X=\bigcup_{i} D\left(2^{i}, 3^{i}, 4^{i}, \ldots\right)$. (Note that $X$ is then $\tau(G)$ from Example 4.8.) Let $Y=\bigcup_{i} D\left((2 !)^{i},(3 !)^{i},(4 !)^{i}, \ldots\right)$. Then $Y$ is an $l$-subgroup of $\prod_{i} \mathscr{R}_{i}$ for the same reasons that $X$ is. 
Now $X$ is lattice isomorphic to $Y$. To prove this, for each $i$, there exists an order permutation $\sigma_{i}$ of $\mathscr{R}_{i}$ such that $\sigma_{i}\left((i+1)^{k}\right)=[(i+1) !]^{k}$. Let $\sigma$ be the lattice automorphism of $\prod_{i} \mathscr{R}_{i}$ determined by the $\sigma_{i}$ 's. Then

$$
\begin{gathered}
\sigma(1,1,1, \ldots)=(1,1,1, \ldots), \sigma(2,3,4, \ldots)=(2 !, 3 !, 4 !, \ldots), \\
\sigma\left(2^{2}, 3^{2}, 4^{2}, \ldots\right)=\left((2 !)^{2},(3 !)^{2},(4 !)^{2}, \ldots\right), \text { etc. }
\end{gathered}
$$

Now suppose by way of contradiction that there exists an $l$-isomorphism $\beta$ mapping $X$ onto $Y$. Without loss of generality, for each $i, \mathscr{R}_{i} \beta=\mathscr{R}_{i}$; so extending $\beta$ to an $l$-automorphism $\beta$ of $D$ means we can assume that $\beta$ is multiplication by a positive element $y \in Y$. But then there exists an integer $n$ such that $y \in D\left((2 !)^{n},(3 !)^{n}, \ldots\right)$ and we can assume that $y<$ $\left((2 !)^{n},(3 !)^{n}, \ldots\right)$ for this $n$.

Now for each integer $k$, there exists an $x \in X$ such that $x \beta>\left((2 !)^{k},(3 !)^{k}\right.$, $\left.(4 !)^{k}, \ldots\right)$ and there exists an $m$ such that $x<\left(2^{m}, 3^{m}, 4^{m}, \ldots\right)$. Therefore, $\left((2 !)^{n} 2^{m},(3 !)^{n} 3^{m}, \ldots\right)>\left(2^{m}, 3^{m}, \ldots\right) y>x y=x \beta>\left((2 !)^{k},(3 !)^{k}, \ldots\right)$. Let $k=2 n$. Then for any integer $j,(j !)^{n} j^{m} \leq(j !)^{2 n 2 m}$; so $j^{m}>(j !)^{n}$ for all $j$ with $m$ and $n$ fixed. But we can select $j$ so that $j^{m}<j$ !. Thus we have a contradiction.

Once again, every example of an l-group with essentially only one addition in this section has been archimedean. We now give a construction that, for any nonzero Specker group $A$, gives a nonarchimedean $l$-group which has essentially only one addition. From this construction, it is easy to build other examples. However, every example we know is abelian and it is an open question if every $l$-group with essentially one addition must be abelian.

So let $(0) \neq A$ be a Specker group and let $G=A \times A$. Since Specker groups are hyperarchimedean, for any two elements $a$ and $b$ of $A, b=b_{a}+b_{a^{\prime}}$, where $b_{a} \in a^{\prime \prime}$ and $b_{a^{\prime}} \in a^{\prime}$. For $(a, b) \in G$, define $(a, b) \geq(0,0)$ if $a \geq 0$ and $b_{a^{\prime}} \geq 0$. Then with componentwise addition,$+(G, \leq,+)$ is an l-group and for any $(x, y) \in G,(x, y) \vee(0,0)=\left(x^{+},\left(x^{+} \vee y^{+}\right)-\left(x^{+} \vee y^{-}\right)+\left(y_{x^{\prime}} \vee 0\right)\right)$ [1].

Proposition 4.10. $(G, \leq)$ has essentially only one addition.

Proof. Let $A_{u}=A \times\{0\}$ and $A_{l}=\{0\} \times A$; then $A_{l}$ is the Specker kernel of $(G, \leq,+)$ and hence of any other addition $\oplus$. Also, $(G, \leq, \oplus) / A_{l}=$ $(G, \leq,+) / A_{l} \cong A$ as lattices, with singular elements of the form $(s, 0)+A_{l}$, where $s$ is singular in $A$. Let $S$ be the set of singular elements of $A$ and let $T=\{(s, 0),(0, s): s \in S\}$. Then $T$ generates $(G, \leq, \oplus)$. So to first prove that $(G, \leq, \oplus)$ is abelian, it suffices to show any two elements of $T$ commute.

Clearly if $x=(0, s)$ and $y=(0, t)$, then $x \oplus y=y \oplus x$. So let $x=(s, 0)$ and $y=(0, t)$. Then there exist $a, b$, and $c$ in $S$ such that $s=a \vee b ; t=$ $b \vee c$; and $a, b$, and $c$ are pairwise disjoint. Then $x \oplus y \ominus x=(a, 0) \oplus(b, 0) \oplus$ $(0, b) \oplus(0, c) \ominus(b, 0) \ominus(a, 0)=(b, 0) \oplus(0, b) \ominus(b, 0) \oplus(0, c)$ since disjoint elements commute. Now $(b, 0) \oplus(0, b) \ominus(b, 0)$ is singular in $(b, 0)^{\prime \prime}=(0, b)^{\prime \prime}$ and so is less than $(0, b)$. Likewise, $\ominus(b, 0) \oplus(0, b) \oplus(b, 0) \leq(0, b)$ and so $(b, 0)$ commutes with $(0, b)$. Thus $x \oplus y \ominus x=y$. A similar argument works if $x=(s, 0)$ and $y=(t, 0)$. Hence $(G, \leq, \oplus)$ is abelian.

Let $n^{*}(b, 0)$ denote the sum of $n(b, 0)$ 's in $(G, \leq, \oplus)$ and $n(b, 0)$ the sum in $(G, \leq,+)$. Then $n^{*}(b, 0) \oplus A_{l}=n(b, 0)+A_{l}$ and so $n^{*}(b, 0)=(n b, x)$ 
for some $x \in b^{\prime \prime}$ in $A$. Thus if $B$ is the subgroup of $(G \leq, \oplus)$ generated by $\{(s, 0): s \in S\}, B$ is an $l$-subgroup of $(G, \leq, \oplus), B \cap A_{l}=(0)$, and for any $(x, y) \in B$ and $(h, k) \in A_{l},|(x, y)| \wedge|(h, k)| \ll|(x, y)|$. So $(G, \leq, \oplus)$ is the direct product of $B$ and $A_{l}$ [1], and $B \cong A_{l}$. But then since $A$ is a free abelian group on some subset of $S$ [23], $(G, \leq, \oplus)$ is free abelian on some subset of $T$. Thus the identity map on $T$ lifts to a group isomorphism $\sigma$ of $(G, \leq, \oplus)$ onto $(G, \leq,+)$.

But $\sigma$ is also an $l$-isomorphism. For if $g \in B, g \sigma$ is the projection of $g$ onto $A_{u}$ and in fact $\sigma$ induces an $l$-isomorphism of $B$ onto $A_{u}$. Thus for any $b \in B$ and $a \in A_{l},(b \sigma)^{+}=b^{+} \sigma,(b \sigma)^{\prime}=b^{\prime}$, and $b \wedge a=b \sigma \wedge a$. So for any $g=b \oplus a \in B \times A_{l},(g \vee 0) \sigma=\left[b^{+} \oplus\left(b^{+} \wedge a^{+}\right) \ominus\left(b^{+} \wedge a^{-}\right) \oplus\left(a_{b^{\prime}} \vee 0\right)\right] \sigma=$ $b^{+} \sigma+\left(b^{+} \wedge a^{+}\right)-\left(b^{+} \wedge a^{-}\right)+\left(a_{b^{\prime}} \vee 0\right)$, while $g \sigma \vee 0=(b \oplus a) \sigma \vee 0=(b \sigma+a) \vee 0=$ $(b \sigma)^{+}+\left((b \sigma)^{+} \wedge a^{+}\right)-\left((b \sigma)^{+} \wedge a^{-}\right)+\left(a_{(b \sigma)^{\prime}} \vee 0\right)$. But then $(g \vee 0) \sigma$ is the same as $g \sigma \vee 0$.

If $A=\mathscr{Z}$, then $(G, \leq,+)=\mathscr{Z} \overleftarrow{\times}$, which is nonarchimedean and so by the above has essentially only one addition. Note that this does not extend to $\mathscr{Z} \overleftarrow{\times} \mathscr{Z} \overleftarrow{\times} \mathscr{Z}$, as on that chain we can define $(a, b, c)(x, y, z)$ to equal $(a+x+c y, b+y, c+z)$ and get a nonabelian nilpotent $o$-group.

\section{LATTICE-ORDERED GROUPS WITH ESSENTIALLY ONLY ONE ABELIAN OR ARCHIMEDEAN ADDITION}

The following theorem from [12] and a subsequent version replacing $\mathscr{R}$ by $\mathscr{Z}$ will be the keys to the main theorem of this section.

Theorem 5.1. If $G$ is a special-valued abelian l-group with $\Delta$ being the minimal plenary subset of $\Gamma(G)$; if $\Delta$ satisfies the descending chain condition; and if for all $\delta \in \Delta, G^{\delta} / G_{\delta} \cong \mathscr{R}$, then $G$ can be l-embedded into $\mathscr{V}(\Delta, \mathscr{R})$ such that $\Sigma(\Delta, \mathscr{R}) \subseteq G$. Moreover, if $G$ is finite-valued, then $G \cong \Sigma(\Delta, \mathscr{R})$.

We now prove that this is true when $\mathscr{R}$ is replaced by $\mathscr{Z}$.

Theorem 5.2. If $G$ is a special-valued abelian l-group with $\Delta$ being the minimal plenary subset of $\Gamma(G)$; if $\Delta$ satisfies the descending chain condition; and if for all $\delta \in \Delta, G^{\delta} / G_{\delta} \cong \mathscr{Z}$, then $G$ can be l-embedded into $\mathscr{V}(\Delta, \mathscr{Z})$ such that $\Sigma(\Delta, \mathscr{Z}) \subseteq G$. Moreover, if $G$ is finite-valued, then $G \cong \Sigma(\Delta, \mathscr{Z})$.

Proof. For each $\delta \in \Delta$, pick a positive special element $g_{\delta} \in G^{\delta} / G_{\delta}$ so that $G_{\delta}+g_{\delta}$ generates $G^{\delta} / G_{\delta}$. It is easily seen that the subgroup $H$ of $G$ generated by the set $\left\{g_{\delta}: \delta \in \Delta\right\}$ is free abelian on $\Delta$ and is an $l$-subgroup $l$-isomorphic to $\Sigma(\Delta, \mathscr{Z})$. Thus the map taking $g_{\delta}$ to the characteristic function of $\{\delta\}$ in $\mathscr{V}(\Delta, \mathscr{Z})$ lifts to an $l$-isomorphism $\sigma$ of $H$ onto $\Sigma(\Delta, \mathscr{Z})$. By Wolfenstein's proof [27] of the Conrad-Harvey-Holland Theorem , $\sigma$ lifts to an $l$-embedding $\sigma$ of $G$ into $\mathscr{V}(\Delta, \mathscr{Q})$ with $\Sigma(\Delta, \mathscr{Z}) \subseteq G \sigma$.

Let $s$ be special in $G$ with value $G_{\delta}$. Then there exists an integer $n$ so that $s-n g_{\delta} \in G_{\delta}$ and so $(s \sigma)_{\delta}=n \in \mathscr{Z}$. Now for any root $\mathscr{C}$ of $\Delta$ through $\delta$, since $\mathscr{C}$ is well ordered, we can assume by induction that for any $\gamma \in \Delta$ with $\gamma<\delta,(s \sigma)_{\gamma} \in \mathscr{Z}$. Thus $s \sigma \in \mathscr{V}(\Delta, \mathscr{Z})$ and so $G \sigma \subseteq \mathscr{V}(\Delta, \mathscr{Z})$.

If $G$ is finite-valued, then for any special element $s$ with value $G_{\delta}$ and integer $n$ such that $s-n g_{\delta} \in G_{\delta}$, again by induction each special component of $\left(s-n g_{\delta}\right) \sigma$ is in $\Sigma(\Delta, \mathscr{Z})$ and so $s \sigma \in \Sigma(\Delta, \mathscr{Z})$. So $G \sigma=\Sigma(\Delta, \mathscr{Z})$. 
Proposition 5.3. If $\Delta$ is a root system satisfying the descending chain condition, then $\Sigma(\Delta, \mathscr{Z}), \Sigma(\Delta, \mathscr{R}), \mathscr{V}(\Delta, \mathscr{Z})$, and $\mathscr{V}(\Delta, \mathscr{R})$ have essentially only one abelian addition.

Proof. We will do this only for $\mathscr{Z}$. From Theorem $2.1, \Sigma(\Delta, \mathscr{Z})$ remains finite-valued and $\mathscr{V}(\Delta, \mathscr{Z})$ remains special-valued under any new additions. From Theorem 2.17, we keep the same set of special values and special covers. Thus under any new abelian addition $\oplus,(\Sigma(\Delta, \mathscr{Z}), \oplus)$ must be $l$-isomorphic to $\Sigma(\Delta, \mathscr{Z})$ with the usual addition.

For $\mathscr{V}(\Delta, \mathscr{Z})$, since $\Delta$ satisfies the descending chain condition, $\mathscr{V}(\Delta, \mathscr{Z})=$ $\Sigma(\Delta, \mathscr{Z})^{\mathscr{L}}$ [12]. Let $(G, \leq, \oplus)$ be $\mathscr{V}(\Delta, \mathscr{Z})$ with a new abelian addition; then without loss of generality, $\Sigma(\Delta, \mathscr{Z}) \subseteq(G \leq, \oplus) \subseteq \mathscr{V}(\Delta, \mathscr{Z})$ and so $(G, \leq,+)^{L}=\mathscr{V}(\Delta, \mathscr{Z})$. But $(G, \leq, \oplus)$ is already laterally complete.

Remark. The descending chain condition is necessary. Charles Holland has communicated to us a proof that $\mathscr{V}(\mathscr{Z}, \mathscr{R})$ is order-isomorphic to $\mathscr{V}\left(\mathscr{Z}^{-}, \mathscr{R}\right)$ as ordered sets. Before his proof, we knew (as said earlier) that $\Sigma(\mathscr{Z}, \mathscr{Z})$ is order-isomorphic to $(\mathscr{Q}, \leq)$.

For the rest of this section, let $A r$ be the class of all $l$-groups with essentially only one archimedean addition and $A b$ be the class of all $l$-groups with essentially only one abelian addition.

Proposition 5.4. Both $A r$ and $A b$ are closed with respect to cardinal sums and products.

Proof. Suppose that $G=A \boxplus B$, where $A, B \in A r$ and suppose that $(G, \leq, \oplus)$ is an archimedean l-group. Then $(G, \oplus)=(A, \oplus) \boxplus(B, \oplus)$ and so is $l$ isomorphic to $(G,+)$.

Now suppose that $\left\{A_{\lambda}\right\}_{\lambda \in \Lambda} \subseteq A r$. Then $\sum_{\Lambda} A_{\lambda} \in A r$. For all $0 \leq g \in$ $\prod_{\Lambda} A_{\lambda}, g=\bigvee a_{\lambda}$ where $a_{\lambda} \in A_{\lambda}$ and if $0<h=\bigvee b_{\lambda} \in \prod_{\Lambda} A_{\lambda}, g+h=$ $\mathrm{V}\left(a_{\lambda}+b_{\lambda}\right)$.

Now each $A_{\lambda}$ remains a cardinal summand of $\left(\prod_{\Lambda} A_{\lambda}, \leq, \oplus\right)$ for any archimedean addition $\oplus$ and so $\left(A_{\lambda},+\right) \cong\left(A_{\lambda}, \oplus\right)$ by way of some $l$-isomorphism $\tau_{\lambda}$. Define $\tau:\left(\prod_{\Lambda} A_{\lambda}, \leq,+\right) \rightarrow\left(\prod_{\Lambda} A_{\lambda}, \leq, \oplus\right): \bigvee a_{\lambda} \rightarrow \bigvee\left(a_{\lambda} \tau_{\lambda}\right)$. Then for all $0 \leq g, h$ in $\left(\prod_{\Lambda} A_{\lambda}, \leq,+\right),(g+h) \tau=\left[\bigvee\left(g_{\lambda}+h_{\lambda}\right)\right] \tau=\bigvee\left(g \tau_{\lambda}+h \tau_{\lambda}\right)=g \tau \oplus h \tau$.

Similar arguments work for $A b$.

We have not been able to show that when $A$ is a cardinal summand of $G \in A b$, then $A \in A b$; in fact, this is not yet resolved even in the case when $A$ is a maximal convex $o$-subgroup. We remark here that such convex $o$-subgroups are double polars of basic elements and so are recognizable from the lattice and the identity. Such an $A$ would of course be an indecomposable summand.

However, if $G \in A b$ and $G=\sum_{\Lambda} A_{\lambda}$ where each $A_{\lambda}$ is indecomposable, we get the desired result.

Proposition 5.5. Suppose $(G, \leq,+) \in A b$ or $A r$, and $G=\sum_{\Lambda} A_{\lambda}$, where each $A_{\lambda}$ is an indecomposable summand of $G$. Then each $A_{\lambda} \in A b$ or $A r$, respectively.

Proof. Choose $\alpha \in \Lambda$ and let $\Delta=\left\{\lambda \in \Lambda: A_{\lambda}\right.$ is lattice isomorphic to $\left.A_{\alpha}\right\}$. Suppose $\oplus$ is a nonisomorphic abelian addition on $A_{\alpha}$. Then $\oplus$ defines a new addition $\oplus$ on $(G, \leq)$ by, for all $\delta \in \Delta,\left(A_{\delta}, \oplus\right) \cong\left(A_{\alpha}, \oplus\right)$, while for 
each $\lambda \in \Lambda \backslash \Delta,\left(A_{\lambda}, \oplus\right)=\left(A_{\lambda},+\right)$. Then $(G, \leq, \oplus) \cong(G, \leq,+)$ as abelian l-groups. Since the $A_{\lambda}$ 's are indecomposable, the $l$-isomorphism must take $\sum_{\Delta}\left(A_{\delta}, \oplus\right)$ onto $\sum_{\Delta}\left(A_{\delta},+\right)$. But then the atomic polars, the $A_{\delta}$ 's for $\delta \in \Delta$, must be isomorphic as well.

\section{OPEN QUESTIONS}

Below we list and discuss some of the questions we have not been able to solve.

(1) If $(G, \leq,+)$ is an l-group such that the positive cone has a unique addition, does $(G, \leq)$ have a unique addition?

Here for any $0 \leq g, h \in G, g \oplus h=g+h$ for any two additions $\oplus$ and + on $(G, \leq)$. We can show that $\oplus=+$ if for all $0 \leq g \in G, \ominus g=-g$.

Proposition 6.1. Let $(G, \leq,+)$ and $(G, \leq, \oplus)$ be l-groups such that for all $0 \leq g, h \in G, g+h=g \oplus h$. If for all $0 \leq g \in G,-g=\ominus g$, then $+=\oplus$.

Proof. First, for all $g \in G, g$ can be written uniquely as $g=a-b$, where $a \wedge b=0$. By Proposition 2.2, $-\ominus g=(-\ominus a)+\ominus b=a-b$. Thus for all $g \in G,-g=\ominus g$.

If $g \wedge h \leq 0, g \vee h=(g-h)^{+}+h=(g-h)^{+} \oplus h$, and so $(g-h)^{+}=$ $g \vee h \ominus h=(g \ominus h)^{+}$. The result then is clear.

We can answer Question 1 affirmatively if $G$ is totally ordered.

Proposition 6.2. If $(G, \leq,+)$ is an o-group such that the positive cone has a unique addition, then $(G, \leq)$ has a unique addition.

Proof. First note that if $0<a \ll b$ in $\left(G^{+}, \leq,+\right)$, then

$$
\tau: x \rightarrow \begin{cases}x+a, & \text { if } x \gg a \\ x, & \text { otherwise }\end{cases}
$$

is an order automorphism of $G^{+}$and so we define a new addition $\oplus$ on $G^{+}$ from $\tau$ as before. Note $b \oplus b=2 b+a \neq 2 b$. So $+\neq \oplus$ on $G^{+}$, a contradiction. Thus $(G, \leq,+)$ must be archimedean and so can be embedded into $\mathscr{R}$ [18].

Now suppose that $(G, \leq, \oplus)$ is also and $o$-group. The map $g \rightarrow-\ominus g$ is a semigroun automorphism of $G^{+}$, for $-\ominus(g+h)=-\ominus(g \oplus h)=-(\ominus g+\ominus h)=$ $-(\ominus g \oplus \ominus h)=-\ominus g+-\ominus h$, and thus there exists $0<r \in \mathscr{R}$ such that $-\ominus g=r g$ for all $g \in G^{+}$[17]. Note that $G^{+}$is $r$-divisible: for any $g \in G^{+}$, there exists $h \in G^{+}$such that $r h=g$.

Without loss of generality, $1+G^{+}$and $r<1$. Define on $G^{+}$

$$
\tau(g)= \begin{cases}r g, & \text { if } 0 \leq g \leq \tau \\ (r+1) g-r, & \text { if } r<g \leq 1 \\ g, & \text { if } g>1\end{cases}
$$

$\tau$ is then an order automorphism of $G^{+}$that is not the identity and is not a homomorphism.

(2) If $G$ is an l-group with unique addition, must $G$ be archimedean?

(3) Does each subdirect product of Ohkuma groups have a unique addition?

(4) Is the class of l-groups with unique addition closed with respect to l-ideals? 
(5) (Kopytov) If $G$ is an l-group with essentially only addition, must $G$ be abelian?

As mentioned before, for a nonabelian $l$-group $(G, \leq,+)$, defining $g \oplus h$ to equal $h+g$ makes $(G, \leq, \oplus)$ into an $l$-group. Thus if $(G, \leq)$ has essentially only one addition, $(G, \leq, \oplus) \cong(G, \leq,+)$.

Andrew Glass has suggested that $(G, \leq,+)=\mathscr{A}(\mathscr{R})$ may be a possible counterexample. Giraudet [21] has shown that if $\oplus$ is an addition on $(G, \leq)$ so that $(G, \leq, \oplus)$ is $l$-isomorphic to an $l$-subgroup $A$ of $\mathscr{A}(T)$ for some chain $T$ so that $A$ acts doubly transitively on $T$, then $(G, \leq, \oplus) \cong \mathscr{A}(\mathscr{R})$. One of us (Darnel) has recently shown that for any $x \in \mathscr{R}$, the point stabilizer $S_{x}=\{g \in \mathscr{A}(\mathscr{R}): g(x)=x\}$ is a maximal convex $l$-subgroup in any addition on $(\mathscr{A}(\mathscr{R}), \leq)$. This means that no normal-valued operation can be placed on $(\mathscr{A}(\mathscr{R}), \leq)$.

(6) If $(G, \leq,+)$ is an archimedean vector lattice, must $(G, \leq, \oplus)$ be archimedean for any other addition $\oplus$ ?

(7) If $(G, \leq,+)$ is hyperarchimedean and $(G, \leq, \oplus)$ is archimedean, must $(G, \leq, \oplus)$ be hyperarchimedean?

(8) Are the classes $\mathrm{Ar}$ and $\mathrm{Ab}$ of l-groups with essentially only one archimedean addition and abelian addition, respectively, closed with respect to cardinal summands?

(9) If $(G, \leq,+)$ is normal-valued and $\oplus$ is another addition for $(G, \leq)$, must $(G, \leq, \oplus)$ be normal-valued?

\section{ADDED IN PROOF}

Questions 1 and 2 have been answered in the affirmative by Jakubik in "On lattice-ordered groups having a unique addition," Czechoslovak Math. J. 40 (115) (1990), 311-314, and in Lattice-ordered groups with unique addition must be Archimedean, Czechoslovak Math. J. 41 (1991). Question 5 has been answered in the negative by Darnel, Giraudet, and McCleary, in Lattice-ordered groups with one essential operation, submitted (1991) to Algebra Universalis. In this paper, it was indeed shown that $\mathscr{A}(\mathscr{R})$ does have essentially only one operation.

\section{REFERENCES}

1. R. Ball, P. Conrad, and M. Darnel, Above and below subgroups of a lattice-ordered group, Trans. Amer. Math. Soc. 297 (1987), 1-40.

2. S. Bernau, Unique representation of lattice groups and normal archimedean lattice rings, Proc. London Math. Soc. 15 (1965), 599-631.

3. A. Bigard, K. Keimel, and S. Wolfenstein, Groupes et anneaux réticulés, Lecture Notes in Math., vol. 608, Springer, 1979.

4. J. P. Bixler and M. Darnel, Special-valued l-groups, Algebra Universalis 22 (1986), 172191.

5. R. Byrd, P. Conrad, and J. Lloyd, Characteristic subgroups of lattice-ordered groups, Trans. Amer. Math. Soc. 158 (1971), 339-371.

6. P. Conrad, Lattice-ordered groups, Tulane Lecture Notes, Tulane Univ., 1970.

7. _ The essential closure of an archimedean lattice-ordered group, Duke Math. J. 38 (1971), 151-160.

8. __ Epi-archimedean groups, Czechoslovak Math. J. 24 (1974), 1-27. 
9. P. Conrad and M. Darnel, l-groups with a unique addition, Algebra and Order, Research and Exposition in Math. 14, Heldermann, 1986, pp. 19-27.

10. P. Conrad and J. Diem, The ring of polar-preserving endomorphisms of an abelian latticeordered group, Illinois J. Math. 15 (1971), 222-240.

11. P. Conrad and D. McAlister, The completion of a lattice-ordered group, J. Austral. Math. Soc. 9 (1969), 182-208.

12. P. Conrad and P. McCarthy, The structure of $f$-algebras, Math. Nachr. 58 (1973), 169-191.

13. M. Darnel, Lattice-ordered groups, Thesis, Univ. of Kansas, 1983.

14. L. Fuchs, Partially ordered algebraic structures, Pergamon Press, 1963.

15. A. M. W. Glass, Ordered permutation groups, London Math. Soc. Lecture Notes Series 55, Cambridge Univ. Press, 1981.

16. A. M. W. Glass, Y. Gurevich, W. C. Holland, and S. Shelah, Rigid homogeneous chains, Math. Proc. Cambridge Philos. Soc. 89 (1981), 7-17.

17. Ja. Hion, Archimedean ordered rings, Uspekhi Mat. Nauk 9:4 (1954), 237-242.

18. O. Hölder, Die Axiome der Quantität und die Lehre vom $M \alpha \beta$, Ber. Verh. Sachs. Wiss. Leipzig, Math-Phys. Cl. 53 (1901), 1-64.

19. W. C. Holland, Transitive lattice ordered permutation groups, Math. Z. 87 (1965), 420-433.

20. J. Jakubik, Representations and extensions of l-groups, Czechoslovak Math. J. 13 (1963), 267-283.

21. M. Jambu-Giraudet, Bi-interpretable groups and lattices, Trans. Amer. Math. Soc. 278 (1983), 253-269.

22. L. Lavis, Sur les quotients totalements ordonnés d'un groupe linéairement ordonné, Bull. Soc. Royal Sci. Liege 32 (1963), 204-208.

23. C. Nobeling, Verallgemeinerung einer Satzes von Hern E. Specker, Invent. Math. 6 (1968), 41-55.

24. T. Ohkuma, Sur quelques ensembles ordonnés linéairement, Fund. Math. 43 (1955), 326337.

25. F. Sik, Zur theory den halbgeordneten gruppen, Czechoslovak Math. J. 6 (1956), 1-25.

26. E. Weinberg, Free lattice-ordered groups. II, Math. Ann. 159 (1965), 217-222.

27. S. Wolfenstein, Sur les groupes reitculés archimédiennement complets, C.R. Acad. Sci. Paris 262 (1966), A813-A816.

Department of MATHEMATICS, UNiversity OF KANSAS, LAWRENCE, KANSAS 66645 46634

Department of Mathematics, Indiana University at South Bend, South Bend, Indiana

Current address: Department of Mathematics and Statistics, Bowling Green State University, Bowling Green, Ohio 43483-0221 